Draft Version November 26, 2015

Preprint typeset using $\mathrm{LT}_{\mathrm{E}} \mathrm{X}$ style emulateapj v. 5/2/11

\title{
HORSESHOE DRAG IN THREE-DIMENSIONAL GLOBALLY ISOTHERMAL DISKS
}

\author{
F. S. MASSET ${ }^{1}$ \\ Instituto de Ciencias Físicas, Universidad Nacional Autónoma de México (UNAM), Apdo. Postal 48-3, 62251-Cuernavaca, Morelos, México \\ P. BeníteZ-LlambaY ${ }^{2}$ \\ Instituto de Astronomía Teórica y Experimental, IATE (CONICET), Observatorio Astronómico, Universidad Nacional de Córdoba, Laprida 854, X5000BGR,
Córdoba, Argentina
Draft version November 26, 2015
}

\begin{abstract}
We study the horseshoe dynamics of a low-mass planet in a three-dimensional, globally isothermal, inviscid disk. We find, as reported in previous work, that the boundaries of the horseshoe region (separatrix sheets) have cylindrical symmetry about the disk's rotation axis. We interpret this feature as arising from the fact that the whole separatrix sheets have a unique value of Bernoulli's constant, and that this constant does not depend on altitude, but only on the cylindrical radius, in barotropic disks. We next derive an expression for the torque exerted by the horseshoe region onto the planet, or horseshoe drag. Potential vorticity is not materially conserved as in two-dimensional flows, but it obeys a slightly more general conservation law (Ertel's theorem) which allows to obtain an expression for the horseshoe drag identical to the expression in a two-dimensional disk. Our results are illustrated and validated by three-dimensional numerical simulations. The horseshoe region is found to be slightly more narrow than previously extrapolated from two-dimensional analyses with a suitable softening length of the potential. We discuss the implications of our results for the saturation of the corotation torque, and the possible connection to the flow at the Bondi scale, which the present analysis does not resolve.

Subject headings: Planetary systems: formation - planetary systems: protoplanetary disks - Accretion, accretion disks — Methods: numerical — Hydrodynamics — Planet-disk interactions
\end{abstract}

\section{INTRODUCTION}

The tide between a low-mass protoplanet and a gaseous protoplanetary disk features two components: the Lindblad torque, which arises from the spiral wake that the embedded planet excites in the disk (Ward 1986, Ogilvie \& Lubow 2002), and the corotation torque, which comes from material slowly drifting near the orbit. In the linear regime, the corotation torque in two-dimensional disks scales with the disk's vortensity ${ }^{2}$ gradient (Tanaka et al. 2002, hereafter TTW02). In inviscid, two-dimensional disks, the corotation torque has been found to always become non-linear, regardless of the planet mass (Paardekooper \& Papaloizou 2009a), and to coincide in this regime with the horseshoe drag (Ward 1991). In two-dimensional barotropic disks, the magnitude of the horseshoe drag scales also with the vortensity gradient $(\mathrm{Ca}-$ soli \& Masset 2009). This dependence is a consequence of the material conservation of vortensity in an inviscid, twodimensional, barotropic flow.

Much less is known of the corotation torque in threedimensional disks. The linear corotation torque has been derived semi-analytically by TTW02 in three-dimensional, globally isothermal disks. They find that it nearly scales with the vortensity gradient. However, no horseshoe drag expression has yet been established in three dimensions, and so far it is unknown whether it would scale with the vortensity gradient.

The purpose of this work is to provide a first step toward

\footnotetext{
masset@icf.unam.mx pbilambay@oac.unc.edu.ar

${ }^{1}$ Send offprint requests to masset@icf.unam.mx

2 The ratio of the vertical component of the flow's vorticity to the surface density.
}

a better understanding of the horseshoe dynamics in three dimensions, and the torque exerted by the horseshoe region on the planet. In a recent work, Fung et al. (2015) have found that the horseshoe flow around a nearly thermal mass planet is columnar: the shape of the outer and inner boundaries of the horseshoe region is nearly cylindrical, and the width of the horseshoe region barely depends on altitude. Lega et al. (2015), using numerical simulations that include sophisticated physics (such as radiative transfer and irradiation of the disk photosphere), have found that the horseshoe region is marginally narrower (by $\sim 10 \%$ ) than in two-dimensional setups with a nominal value of the softening length of the potential. We will compare these findings to our results.

We follow hereafter an approach inspired in Masset et al. (2006) and Casoli \& Masset (2009): we use Bernoulli's invariant to provide useful information about the horseshoe region and to derive a horseshoe drag expression. We limit ourselves here to inviscid, globally isothermal disks, and we focus on the unsaturated horseshoe drag: we assume that a sufficient amount of time has elapsed after the planet insertion for the corotation torque to become non-linear, but we also assume that this time is shorter than the time that separates two consecutive close encounters of a fluid element with the planet, which is usually much longer than the U-turn time scale. While Masset et al. (2006) investigated the transition between low and large mass planets, and found a boost of the horseshoe width (and drag) for intermediate mass, non gapopening planets, we restrict ourselves here to the low-mass case, for which the width of the horseshoe region scales as the square root of the planetary mass.

Our paper is organized as follows: in section 2 we define our notation and give the governing equations of the problem. 
In section 3, we discuss some topological properties of the horseshoe region, and we derive a horseshoe drag expression valid exclusively in globally isothermal disks, using Ertel's potential vorticity theorem (Ertel 1942). This derivation allows us to give an adequate definition of the vortensity gradient in three-dimensional disks. In section 4 , we describe the numerical code and the setup used in the numerical simulations that we performed to check and illustrate the properties of section 3 In sections 5 and 6 we summarize and discuss our results.

\section{NOTATION \& EQUATIONS}

\subsection{Notation}

We study the tidal interaction between a gaseous globally isothermal (hence barotropic) protoplanetary disk in rotational equilibrium around a star of mass $M_{\star}$, and an embedded protoplanet of mass $M_{p}=q M_{\star}$, on a fixed circular orbit in the disk's midplane, with orbital radius $r_{p}$ and orbital frequency $\Omega_{p}$. We give the position of a given fluid element either in spherical coordinates $(r, \theta, \phi)$ in a frame corotating with the planet, or in cylindrical coordinates, $R=r \sin \theta$ being the cylindrical radius and $z=r \cos \theta$ being the altitude. In each case the frame is centered on the star and such that the planet's $\phi$-coordinate equal zero. The angular speed of a fluid element in a non-rotating frame is denoted $\Omega(r, \theta, \phi)$. We denote with a 0 subscript a quantity in the unperturbed disk (i.e. prior to the insertion of the planet), and with a $p$ subscript a quantity evaluated at $r=r_{p}$. The surface density is defined as

$$
\Sigma(R)=\int_{-\infty}^{+\infty} \rho(R, z) d z
$$

where $\rho$ is the volumic density. In the unperturbed disk, its midplane value follows a power law:

$$
\rho(r, \theta=\pi / 2, \phi) \propto r^{-\xi} \text {. }
$$

The (uniform) soundspeed is denoted $c_{s}$, the pressure scale height is $H(r)=c_{s} \sqrt{r^{3} / G M_{\star}}$, and the disk aspect ratio is $h(r)=H(r) / r$. Since the disk is globally isothermal, the aspect ratio increases as the square root of the radius: $h(r)=$ $h_{p}\left(r / r_{p}\right)^{1 / 2}$. One can work out the exact dependence of the volumic density and rotational velocity on $r$ and $\theta$, for the case in which the temperature is a power law of the spherical radius, as described in appendix $\mathrm{A}$ From these dependencies, one can infer that the surface density also obeys a power law of radius: $\Sigma \propto r^{-\alpha}$, where $\alpha$ is given by Eq. A23.

We introduce the vorticity of the flow $\zeta$ viewed in an inertial frame:

$$
\zeta=\boldsymbol{\nabla} \times \boldsymbol{v}+2 \mathbf{\Omega}_{p},
$$

where $v$ is the linear velocity in the frame corotating with the planet. Throughout this work, we denote the potential vorticity (PV) with:

$$
w=\frac{\zeta}{\rho}
$$

In order to use a vocabulary consistent with prior work in two dimensions, we reserve the name vortensity for an adequate vertical integral of the PV, which we shall introduce later.

We define the corotation sheet as the two-dimensional region where $\Omega_{0}(r, \theta, \phi)=\Omega_{p}$. This sheet intersects the midplane at $r=r_{c}(\phi)$.

We focus on the horseshoe region, defined by the particles that cross over from the inner to the outer disk, or vice-versa, after a close encounter with the planet. We call the boundary between the horseshoe region and the rest of the disk the separatrix sheet. The streamlines belonging to this sheet are called either the separatrix streamlines, critical streamlines, or widest horseshoes (Fung et al. 2015).

At large azimuthal elongation from the planet, the horseshoe region can be divided into four regions. The front (rear) part corresponds to fluid elements with $\phi>0(\phi<0)$. Inside the front and rear parts, we distinguish the upstream and downstream regions. The upstream region is the set of fluid elements that have not experienced yet a close encounter with the planet, whereas the downstream region is the set of fluid elements that have already experienced a close encounter, and therefore crossed the corotation by definition of the horseshoe region. In the front part, the upstream region corresponds to $r>r_{c}$, and the downstream region to $r<r_{c}$. Opposite relations hold in the rear part.

\subsection{Governing equations}

The equations that govern the evolution of the flow are the continuity equation and the Euler equations, which read respectively, in cylindrical coordinates and in the frame corotating with the planet:

$$
\begin{gathered}
\partial_{t} \rho+\frac{1}{R} \partial_{R}\left(R \rho v_{R}\right)+\frac{1}{R} \partial_{\phi}\left(\rho v_{\phi}\right)+\partial_{z}\left(\rho v_{z}\right)=0, \\
\partial_{t} v_{R}+v_{R} \partial_{R} v_{R}+\frac{v_{\phi}}{R} \partial_{\phi} v_{R}+v_{z} \partial_{z} v_{R} \\
-R \Omega_{p}^{2}-2 \Omega_{p} v_{\phi}-\frac{v_{\phi}^{2}}{R}=-\frac{\partial_{R} P}{\rho}-\partial_{R} \Phi, \\
\partial_{t} j+v_{R} \partial_{R} j+\frac{v_{\phi}}{R} \partial_{\phi} j+v_{z} \partial_{z} j=-\frac{\partial_{\phi} P}{\rho}-\partial_{\phi} \Phi
\end{gathered}
$$

and

$$
\partial_{t} v_{z}+v_{R} \partial_{R} v_{z}+\frac{v_{\phi}}{R} \partial_{\phi} v_{z}+v_{z} \partial_{z} v_{z}=-\frac{\partial_{z} P}{\rho}-\partial_{z} \Phi,
$$

where $v_{R}, v_{\phi}$ and $v_{z}$ are the cylindrical components of the velocity in the rotating frame, $P=\rho c_{s}^{2}$ is the pressure, $j=R^{2} \Omega=R^{2} \Omega_{p}+R v_{\phi}$ is the specific angular momentum, evaluated in the non-rotating frame, and $\Phi$ is the gravitational potential given by:

$$
\begin{array}{r}
\Phi=-\frac{G M_{\star}}{\sqrt{R^{2}+z^{2}}}-\frac{G M_{p}}{\sqrt{R^{2}+r_{p}^{2}-2 R r_{p} \cos \phi+z^{2}}} \\
+\frac{G M_{p}}{r_{p}^{2}} R \cos \phi,
\end{array}
$$

where the different terms are respectively the star potential, the planet potential and the indirect term arising from the acceleration of our non-inertial frame, centered on the star.

\section{UNSATURATED HORSESHOE DRAG}

We make the assumption that the flow in the vicinity of the planet is in steady state, and that the upstream regions are essentially unperturbed. As has been discussed previously (Casoli \& Masset 2009, Masset \& Casoli 2009, Paardekooper et al. 2010), this amounts to considering the flow at a time 
after the insertion of the planet that is larger than the Uturn timescale, but shorter than (half) the horseshoe libration timescale, in order to avoid possible saturation effects. The torque given by our analysis is therefore the unsaturated horseshoe drag: the material that experiences close encounters with the planet is "fresh" material, which has never experienced another close encounter previously.

\subsection{A Bernoulli invariant}

The right hand side of Eqs. (6) to (8) can be recast as the gradient of the effective potential

$$
\tilde{\Phi}=\Phi+\eta
$$

where

$$
\eta=c_{s}^{2} \log \left(\frac{\rho}{\rho_{00}}\right),
$$

is the fluid enthalpy, and $\rho_{00}$ is an arbitrary constant dimensionally homogeneous to a density. Like the gravitational potential, the enthalpy is defined to within an additive constant, and specifying $\rho_{00}$ amounts to choosing for which value of the density the enthalpy vanishes. In what follows we set $\rho_{00}=M_{*} r_{p}^{-3}$. Multiplying Eqs. (6), (7) and (8) respectively by $v_{R}, v_{\phi} / R$ and $v_{z}$, and summing the results under the assumption of a steady flow, we are left with:

$$
\boldsymbol{v} \cdot \boldsymbol{\nabla}\left(E_{\mathrm{kin}}+\tilde{\Phi}\right)-R v_{R} \Omega_{p}^{2}=0,
$$

where $\boldsymbol{v} \cdot \boldsymbol{\nabla} \equiv v_{R} \partial_{R}+\frac{v_{\phi}}{R} \partial_{\phi}+v_{z} \partial_{z}$ and

$$
E_{\mathrm{kin}}=\frac{1}{2}\left(v_{R}^{2}+v_{\phi}^{2}+v_{z}^{2}\right) \text {, }
$$

hence we have:

$$
\boldsymbol{v} \cdot \boldsymbol{\nabla} B_{J}=0
$$

where

$$
B_{J}=E_{\mathrm{kin}}+\tilde{\Phi}-\frac{1}{2} R^{2} \Omega_{p}^{2}
$$

is a Bernoulli invariant. The $J$ index is in analogy with the Jacobi constant of a test particle, and is also meant to avoid confusion with Oort's second constant, that we shall introduce later. This invariant is conserved along the streamlines of the domains over which Eq. (6) to (8) hold, that is to say over the domains that do not contain shocks. We assume this to be the case, in particular, of the coorbital region of sufficiently low mass planets.

\subsection{Considerations about the topology of the horseshoe region}

In previous two-dimensional analyses (Casoli \& Masset 2009, Masset \& Casoli 2009), the width of the horseshoe region far from the planet can be determined using the fact that Bernoulli's invariant is the same at the stagnation point and far away on the separatrices. From Eq. (15) this value of the Bernoulli invariant depends exclusively on the enthalpy of the fluid at the stagnation point and its location. We discuss below whether a similar property can be generalized to the three-dimensional case.

The first question to address is therefore that of the set of stagnation points. A stagnation point, in three dimensions, is found wherever the three components of the velocity simultaneously vanish. In general, the constraint $v_{\phi}=0$ is verified on a two-dimensional manifold (the corotation sheet), within which the additional constraint $v_{R}=0$ yields a onedimensional manifold. Then, in general, the additional constraint $v_{z}=0$ yields a finite number of points within this onedimensional manifold. We therefore expect to have a finite number of stagnation points in three dimensions. The streamline analysis performed on data of three-dimensional calculations, presented in section 4.2 , corroborates this statement.

In the following we shall assume that in the threedimensional case the horseshoe region is still bounded by a well-defined set of streamlines, the separatrix sheets, threedimensional generalization of the separatrix streamlines of the two-dimensional cases. We will come back to this assumption in the discussion of section 6.4. The second question is whether all fluid elements of a separatrix sheet go through a stagnation point. While the answer to this question is trivial in the two-dimensional case, it is not that obvious in the three-dimensional case. In particular, one can imagine that a given fluid element of a separatrix, originating at a given altitude, will complete a horseshoe U-turn without ever reaching the altitude of the stagnation point. Assume that such a fluid element exists. By hypothesis, the norm of its velocity is therefore finite everywhere on its associated streamline 3 Then consider another fluid element on a neighboring streamline, initially close to the first one. The separation $\boldsymbol{\xi}$ between both fluid elements then obeys $d \boldsymbol{\xi} / d t=\boldsymbol{v}_{2}-\boldsymbol{v}_{1}$, where $\boldsymbol{v}_{2}$ and $v_{1}$ are the velocities of both fluid elements along their trajectories. One can recast this derivative in terms of the curvilinear abscissa $s$ along the trajectory of the first fluid element: $d \boldsymbol{\xi} / d s=\left(\boldsymbol{v}_{2}-\boldsymbol{v}_{1}\right) / v_{1}$. Since $v_{1}$ remains finite and since the velocity field is continuous, $d \boldsymbol{\xi} / d s$ can be made arbitrarily small provided the fluid elements are sufficiently close initially. Upon integration over any finite length $s$, this means that two neighboring streamlines can always remain arbitrarily close to each other, provided their initial separation is adequately chosen. This is in contradiction with the character of a separatrix: two neighboring streamlines on opposite sides of the separatrix sheet always follow divergent paths after a finite length, no matter how close they are initially. This shows that the modulus of the velocity vector along a separatrix streamline must vanish somewhere, which implies the passage through a stagnation point. There is an immediate consequence to this statement: the streamlines of the separatrix sheets have significant vertical excursions in order to pass through one of the stagnation points. By continuity, the horseshoe trajectories are vertically bent near the U-turns, especially those which are close to the separatrix. This has already been observed in three-dimensional calculations (D'Angelo et al. 2003; Fung et al. 2015).

There is another consequence to the fact that all separatrices go through at least one of the stagnation points: the value of Bernoulli's invariant on any separatrix streamline must be the value that it has at the corresponding stagnation point. By spawning streamlines (downstream and upstream) in all directions in the immediate vicinity of a stagnation point, one generates a separatrix sheet with a unique value of Bernoulli's invariant.

As in the two-dimensional case, there may be several stagnation points, each with its own value of the Bernoulli invariant. Each of these points generates a separatrix sheet where Bernoulli's invariant has everywhere the same value as at the point. We note that two separatrix sheets with different values

\footnotetext{
${ }^{3}$ This streamline coincides with the path followed by the fluid element, under our assumption of a steady state.
} 
of Bernoulli's invariant cannot cross, so that they are disjoint or nested. We also show in appendix $B$ that a given separatrix sheet cannot be connected to two stagnation points with different values of Bernoulli's invariant.

In what follows, we are interested in the separatrix sheet that lies furthest from the orbit, corresponding to the widest horseshoe streamlines. This is to be compared to the twodimensional situation, which can feature two X-stagnation points (Casoli \& Masset 2009, Guilet et al.2013), but the one that determines the overall horseshoe dynamics is the one that has the lowest value of the Bernoulli invariant (hence connected to streamlines that lie the furthest from the orbit).

An important hypothesis is that the flow is barotropic: all variables of Eq. (15) are continuous in a barotropic fluid, hence the value of Bernoulli's invariant is well-defined in the vicinity of a stagnation point. In a baroclinic situation (if, for instance, the flow obeyed an energy equation), the enthalpy, hence Bernoulli's invariant, would not necessarily be continuous.

The fact that Bernoulli's invariant is uniform on a separatrix sheet provides an idea of the vertical shape of the horseshoe region far from the planet. Considering that the flow at large azimuthal distance from the planet is unperturbed, we can simplify the expression of Bernoulli's invariant given by Eq. (15) as:

$$
B_{J}=\frac{1}{2} v_{\phi}^{2}+\Phi_{*}-\frac{1}{2} R^{2} \Omega_{p}^{2}+c_{s}^{2} \log \left(\frac{\rho_{0}}{\rho_{00}}\right),
$$

where $\Phi_{*}(\boldsymbol{r})$ is the stellar potential. As shown in appendix A the azimuthal velocity in a globally isothermal disk in rotational and hydrostatic equilibrium does not depend on the altitude (Eq. A19), so the vertical derivative of Bernoulli's invariant reduces to:

$$
\partial_{z} B_{J}=\partial_{z} \Phi_{*}+c_{s}^{2} \partial_{z} \log \left(\frac{\rho_{0}}{\rho_{00}}\right) .
$$

The right hand side of Eq. 17 cancels out, as can be seen from Eq. (8) for an axisymmetric disk in equilibrium, hence Bernoulli's invariant in the unperturbed disk depends only on the cylindrical radius. The horseshoe's separatrix sheets should therefore be cylinders coaxial with the disk's rotation axis, and so should the corotation sheet, since $v_{\phi}$ also depends exclusively on the cylindrical radius. The horseshoe region is therefore expected to have a constant width with altitude. This has been seen by Fung et al. (2015), who describe the horseshoe flow as columnar. This property will be corroborated by numerical simulations in section 4 This allows us to introduce unambiguously the (unique) half-width of the horseshoe region, which we denote $x_{s}$ as in previous two-dimensional work.

Note that the cylindrical shape of the corotation sheet is a consequence of the disk being globally isothermal. Expanding in $z$ the relations given in appendix A, we can see that for the case in which the temperature increases outward, the corotation has its smallest radius at the midplane, and the opposite holds for the more realistic case corresponding to a temperature decreasing outward: its largest radius is at the midplane.

\subsection{Torque expression \\ 3.3.1. Domain of interest}

As in Casoli \& Masset (2009) and Masset \& Casoli (2009), we define a domain of interest $\mathcal{D}$ that encloses the planet and the horseshoe region, that extends from $z=-\infty$ to $z=+\infty$, from $\phi=\phi_{-}$to $\phi=\phi_{+}$, where $\phi_{-}<0$ and $\phi_{+}>0$, and where $\left|\phi_{-}\right|$and $\phi_{+}$are chosen sufficiently large so that the planetary potential is negligible at $\phi=\phi_{ \pm}$. Similarly, we chose the radial boundaries of the domain, $R_{\min }$ and $R_{\max }$, to cover an extent much wider than the horseshoe region. Our domain therefore encloses not only horseshoe streamlines, but also streamlines that do not perform a U-turn, and which belong to the inner or outer disk. We make the assumption that the horseshoe flow reaching $\phi_{ \pm}$is relaxed in the sense that the flow velocity is exclusively azimuthal, and the azimuthal derivatives of the hydrodynamic variables vanish. Naturally, a given fluid element starting its horseshoe trajectory at a given altitude $z_{0}$ may change its altitude during its journey to the downstream part of the horseshoe flow. As we shall see in numerical simulations, it may also oscillate vertically a few times after the Uturn. We make no restrictive assumption concerning its final altitude, which may be different from $z_{0}$. Our only assumption at this stage is that its altitude has converged to a constant value by the time it reaches the azimuth $\phi_{ \pm}$, which is why we must consider sufficiently large values of $\left|\phi_{ \pm}\right|$.

The torque exerted by the material enclosed in the domain $\mathcal{D}$ on the planet is:

$$
\Gamma=\iiint_{\mathcal{D}} \rho \partial_{\phi} \Phi R d R d z d \phi .
$$

Using Eqs. (7), then (5), under our assumption of steady state, this integral can be recast as integrals that account for the angular momentum budget on the faces of the domain:

$$
\begin{aligned}
\Gamma= & -\left.\int_{-\infty}^{+\infty} d z \int_{\phi_{-}}^{\phi_{+}} d \phi \rho v_{R} j R\right|_{R_{\max }}+\left.\int_{-\infty}^{+\infty} d z \int_{\phi_{-}}^{\phi_{+}} d \phi \rho v_{R} j R\right|_{R_{\min }} \\
& -\left.\int_{-\infty}^{+\infty} d z \int_{R_{\min }}^{R_{\max }} d R\left(\rho v_{\phi} j+R P\right)\right|_{\phi_{+}} \\
& +\left.\int_{-\infty}^{+\infty} d z \int_{R_{\min }}^{R_{\max }} d R\left(\rho v_{\phi} j+R P\right)\right|_{\phi_{-}}
\end{aligned}
$$

The edge terms in $z$ cancel out since the density vanishes at larger altitude. The integrals of Eq. (19) involve angular momentum fluxes on the arc-of-cylinder faces of the domain (in $R=R_{\min }$ and $R=R_{\max }$ ), and on the radial faces of the domain (in $\phi=\phi_{ \pm}$).

\subsubsection{Corotation torque integral}

We now assume that the angular momentum flux on the arcof-cylinder faces is the flux carried by the spiral wake, which corresponds to the Lindblad torque on the planet, and that the fluxes on the radial faces of the domain correspond, on the contrary, to the corotation torque. We assume that there is a range of aspect ratios $\left(R_{\max }-R_{\min }\right) /\left[r_{p}\left(\phi_{+}-\phi_{-}\right)\right]$of the domain for which this separation of the torques is correct, and we focus exclusively on the flux on the radial faces, which we interpret as the full, non-linear corotation torque:

$$
\begin{aligned}
\Gamma_{\mathrm{CR}}=-\left.\int_{-\infty}^{+\infty} d z \int_{R_{\min }}^{R_{\max }} d R\left(\rho v_{\phi} j+R P\right)\right|_{\phi_{+}} \\
+\left.\int_{-\infty}^{+\infty} d z \int_{R_{\min }}^{R_{\max }} d R\left(\rho v_{\phi} j+R P\right)\right|_{\phi_{-}} .
\end{aligned}
$$


We lay down a last assumption: there is no shock in the domain of interest $\mathcal{D}$. As the latter includes the planetary wake in the vicinity of the planet, this domain should be narrow enough so as not to reach the location at which the wake eventually shocks as a consequence of wave steepening. This location can be dangerously close to the planet owing to the differential rotation of the disk (Goodman \& Rafikov 2001), so we focus on planetary masses largely below the thermal mass. This assumption is required, stricto sensu, to satisfy our steady state assumption. Should shocks be present over the domain, they would transfer the planetary torque to the disk material and they would carve a gap (Rafikov 2002) over a time scale longer than the typical time considered here, intermediate between the horseshoe U-turn and libration time scales, precluding the existence of a steady state. Arguably shocks could still appear beyond the radial limits of the domain $\mathcal{D}$. We assume that radial boundary conditions are tailored to ensure a steady state over $\mathcal{D}$.

Using Eq. (16) and the assumption that the motion is exclusively azimuthal at large azimuthal distances from the planet, we write:

$$
\partial_{R} B_{J}=\zeta_{z} v_{\phi}
$$

and we change our integration variable to $B_{J}$ so as to transform Eq. 20] into:

$$
\begin{aligned}
\Gamma_{\mathrm{CR}}= & -\left.\int_{-\infty}^{+\infty} d z \int_{B_{m}}^{B_{c}} d B_{J} \frac{\rho v_{\phi} j+R P}{\zeta_{z} v_{\phi}}\right|_{\phi_{+}, R<R_{c}} \\
& -\left.\int_{-\infty}^{+\infty} d z \int_{B_{m}}^{B_{c}} d B_{J} \frac{\rho v_{\phi} j+R P}{\zeta_{z} v_{\phi}}\right|_{\phi_{+}, R>R_{c}} \\
& +\left.\int_{-\infty}^{+\infty} d z \int_{B_{m}}^{B_{c}} d B_{J} \frac{\rho v_{\phi} j+R P}{\zeta_{z} v_{\phi}}\right|_{\phi_{-}, R<R_{c}} \\
& +\left.\int_{-\infty}^{+\infty} d z \int_{B_{m}}^{B_{c}} d B_{J} \frac{\rho v_{\phi} j+R P}{\zeta_{z} v_{\phi}}\right|_{\phi_{-}, R>R_{c}},
\end{aligned}
$$

where $B_{m}$ represents the minimum value of Bernoulli's invariant, at larger distance from corotation (without loss of generality we can assume that it has same value at the outer and inner sides), $B_{c}$ represents the value of Bernoulli's invariant at corotation, and $B_{s}$ its value on the separatrix sheet.

Note that the relation between $R$ and $B_{J}$ is not one-to-one. Eq. (21) shows that the radial derivative of Bernoulli's invariant changes sign at corotation, where $B_{J}$ is maximum. We therefore had to split in two each term of Eq. 20, specifying whether we consider contributions from inside or outside corotation. We number one to four, in their order of appearance, the four terms of the right hand side of Eq. 22). Terms one and four correspond to downstream material leaving the domain, while terms two and thee correspond to upstream material entering it. Regardless of the complexity of the flow in the vicinity of the planet, a fluid element entering the domain either leaves it on the same side of corotation, or on the other side.

We examine firstly the case of a fluid element leaving the domain on the same side of corotation ("circulating" rather than "librating"). For the sake of definiteness we assume it enters the domain in $\phi_{+}, R>r_{c}$. It therefore leaves it in $\phi_{-}$, $R>r_{c}$. An example of the stream tube defined by such fluid element is depicted in blue in Fig. 1. The contribution of this

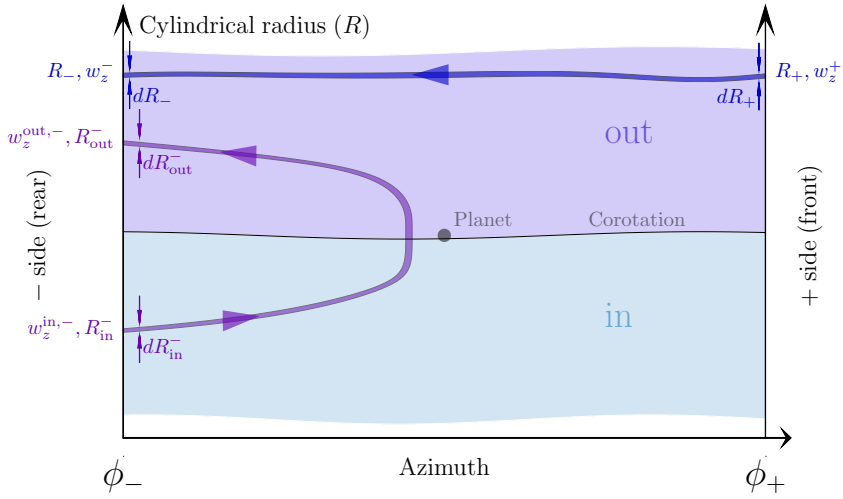

FIG. 1.- Sketch of circulating (blue) and librating (purple) stream tubes. Each tube is associated to a given range $\left[B_{J}, B_{J}+d B_{J}\right]$ of Bernoulli's invariant (different in the two cases). For the sake of legibility, not all the variables used in the derivation in the text are shown, but their meaning and the location at which they are defined are straightforward to infer from the variables shown here. For the same reason, the vertical dimension is not represented on this sketch, which shows a projection on the horizontal plane $(R, \phi)$. It must be kept in mind that the endpoints of the stream tubes may have different altitudes, and different vertical extents.

term to the second integral of Eq. 22, is:

$$
d \Gamma_{\mathrm{CR}}^{+}=-\left.d B_{J} d z_{+} \frac{\rho v_{\phi} j+R P}{\zeta_{z} v_{\phi}}\right|_{\phi_{+}}
$$

and its contribution to the fourth one is:

$$
d \Gamma_{\mathrm{CR}}^{-}=\left.d B_{J} d z_{-} \frac{\rho v_{\phi} j+R P}{\zeta_{z} v_{\phi}}\right|_{\phi_{-}} .
$$

Since we assumed there are no shocks on the domain, the fluid elements have their Bernoulli's invariant materially conserved. We can work out the net flux contribution by summing Eqs. (23) and (24).

The fluid element may exit the domain at a radius and altitude different from those at which it entered the domain, and its velocity and enthalpy may also be different. We assume the variations of radius, velocity and enthalpy to be sufficiently small to allow first order expansions. We denote with $\delta X$ the small variation of a quantity $X$ at the fluid element location, between its arrival to and exit from the domain (i.e. the Lagrangian variation seen by the fluid element), and with $\delta^{\prime} X$ the variation of that quantity between the two radial faces, at given radius and altitude. Expanding Eq. (16), making use of Eqs. (6) and (8) together with the assumptions we laid down at large azimuthal distance from the planet $\left(v_{R}=v_{z}=0\right.$, $\left.\partial_{\phi} \equiv 0\right)$, we are led to:

$$
\delta B_{J}=\left(\frac{v_{\phi}}{R}\right)_{\phi_{-}} \delta j+\delta^{\prime} \eta,
$$

with $\delta j=j_{-}-j_{+}$and $\delta^{\prime} \eta=\eta\left(R, z, \phi_{-}\right)-\eta\left(R, z, \phi_{+}\right)$. Since $\delta B_{J}=0$, we have:

$$
\begin{aligned}
d \Gamma_{\mathrm{CR}}^{-} & =\frac{d B_{J} d z_{-}}{\zeta_{z}^{-}}\left[\rho_{-}\left(j_{+}-\frac{R \delta^{\prime} \eta}{v_{\phi}}\right)+\frac{R P}{v_{\phi}}\right]_{\phi_{-}} \\
& =\frac{d B_{J} d z_{-}}{w_{z}^{-}} j_{+}+d R_{-} d z_{-} R_{-} P\left(R_{-}, z_{-}, \phi_{+}\right),
\end{aligned}
$$

where we have used the first order expansion $\delta^{\prime} P=\rho_{-} \delta^{\prime} \eta$, and where $d R_{ \pm}$is the radial width of the stream tube at azimuth 
$\phi_{ \pm}$. In order to further transform the first term, we use Ertel's theorem ${ }^{4}($ Ertel 1942). Ertel's theorem, in a reduced form that is of interest here, states that in a barotropic flow we have the conservation law:

$$
D_{t}\left(\frac{\zeta}{\rho} \cdot \nabla \psi\right)=0
$$

where $\psi$ is any quantity materially conserved by the flow $\left(D_{t} \psi=0\right)$. We can take here for $\psi$ an arbitrary function whose derivative in $z$ does not vanish, such as $z_{+}$itself, regarded as a Lagrangian, passive scalar on the fluid elements. Ertel's theorem reads in this case:

$$
\left(\boldsymbol{w} \cdot \boldsymbol{\nabla} z_{+}\right)_{\phi_{+}}=\left(\boldsymbol{w} \cdot \boldsymbol{\nabla} z_{+}\right)_{\phi_{-}},
$$

or

$$
w_{z}^{+}=w_{z}^{-} \frac{d z_{+}}{d z_{-}}+w_{R}^{-} \frac{d z_{+}}{d R} .
$$

Note that in Eq. (29) there is no azimuthal derivative, as per our assumption that these vanish at large azimuthal distance from the planet. The factor $w_{R}^{-}$in the last term is expected to be much smaller than $w_{z}^{ \pm}$(we will see in section 4 that the vorticity tilt angle, especially in the circulating region, is well below $10^{-2}$ ), and we expect the second factor of this term $\left(d z_{+} / d R\right.$, which represents the inclination of an initially horizontal sheet of material after the interaction with the planet), to be also a small number. We therefore write:

$$
\frac{d z_{+}}{w_{z}^{+}} \approx \frac{d z_{-}}{w_{z}^{-}}
$$

with an accuracy largely better than the $10^{-2}$ level. The net contribution to the corotation torque of the stream tube that we considered is therefore:

$$
\begin{aligned}
d \Gamma_{\mathrm{CR}}=d \Gamma_{\mathrm{CR}}^{+}+d \Gamma_{\mathrm{CR}}^{-}= & d R_{-} d z_{-} R_{-} P\left(R_{-}, z_{-}, \phi_{+}\right) \\
& -d R_{+} d z_{+} R_{+} P\left(R_{+}, z_{+}, \phi_{+}\right) .
\end{aligned}
$$

We see that the torque contribution of our circulating fluid element only amounts to the pressure on the upstream side: both terms above are evaluated in $\phi_{+}$thanks to the transformation arising from Eq. (25). Anticipating on what follows, this pressure contribution will cancel out upon the integration on all stream tubes, so that the net contribution will exclusively arise from the horseshoe region, which we now examine.

We consider a stream tube that executes a horseshoe U-turn (corresponding to "librating" fluid elements). For the sake of definiteness we assume it enters the domain of interest in $R<r_{c}$ and $\phi_{-}$and exits it in $R>r_{c}$ and $\phi_{-}$, such as the one depicted in purple in Fig. 1. Such stream tube yields contributions to the third and fourth integrals of Eq. 22, which read respectively:

$$
\begin{gathered}
\left.d \Gamma_{\mathrm{CR}}^{-}\right|_{\text {in }}=\left.d B_{J} d z_{-} \frac{\rho v_{\phi} j+R P}{\zeta_{z} v_{\phi}}\right|_{\phi_{-}, \text {in }} \\
\left.d \Gamma_{\mathrm{CR}}^{-}\right|_{\text {out }}=\left.d B_{J} d z_{-} \frac{\rho v_{\phi} j+R P}{\zeta_{z} v_{\phi}}\right|_{\phi_{-}, \text {out }},
\end{gathered}
$$

where the subscript in or out specifies whether we consider a region inside or outside corotation. In the above analysis of a circulating stream tube, from Eq. 23, to Eq. (31), fluid

${ }^{4}$ The original paper of Ertel has been translated to English by Schubert et al. (2004). elements did not cross corotation, so there was no need to specify explicitly the location of each term as they were all evaluated systematically outside corotation. As previously, we can recast Eq. (32) as:

$$
\left.d \Gamma_{\mathrm{CR}}^{-}\right|_{\text {in }}=\frac{d B_{J} d z_{\text {in }}^{-}}{w_{z}^{\text {in,-- }}} j_{\text {in }}^{-}-d R_{\text {in }}^{-} d z_{\text {in }}^{-} R_{\text {in }}^{-} P\left(\phi_{-}, R_{\text {in }}^{-}, z_{\text {in }}^{-}\right) .
$$

We can write Eq. (33) in a similar way, and transform it using Eq. 25], for which we set $\delta B_{J}=0$ :

$$
\begin{aligned}
\left.d \Gamma_{\mathrm{CR}}^{-}\right|_{\text {out }} & =\frac{d B_{J} d z_{\text {out }}^{-}}{w_{z}^{\text {out },-}} j_{\text {out }}^{-}+d R_{\text {out }}^{-} d z_{\text {out }}^{-} R_{\text {out }}^{-} P\left(\phi_{-}, R_{\text {out }}, z_{\text {out }}\right) \\
& =\frac{d B_{J} d z_{\text {out }}^{-}}{w_{z}^{\text {out },-}} j_{\text {out }}^{+}+d R_{\text {out }}^{-} d z_{\text {out }}^{-} R_{\text {out }}^{-} P\left(\phi_{+}, R_{\text {out }}, z_{\text {out }}\right)(35)
\end{aligned}
$$

We see that this transformation allows to express the torque contribution in term of the upstream pressure $P\left(\phi_{+}, R_{\text {out }}^{-}, z_{\text {out }}^{-}\right)$ and specific angular momentum $j_{\text {out }}^{+}$on the outer side. Since the transformation of Eq. (35) has been obtained by letting $\delta B_{J}=0$, the quantity $j_{\text {out }}^{+}$represents the specific angular momentum of the upstream material in the outer disk that has same Bernoulli's invariant as our horseshoe stream tube.

When summing all contributions of Eq. (22), it is now clear that the contributions of the pressure torques cancel out, since they all amount to summing the upstream pressure field: in the outer disk, Eq. 31 for the circulating fluid elements and Eq. (35) for the librating ones show that we only consider the upstream pressure $\left(P\left(\phi_{+}\right)\right)$. For the inner disk transformations similar to those considered previously would yield torque contributions as function of $P\left(\phi_{-}\right)$, such as the contribution given by Eq. (32). Since the angular momentum advective contributions cancel out for circulating streamlines, as shown by Eq. (31), we are only left with the contributions to the angular momentum flux of the librating streamlines. For the rear horseshoe, the total contribution therefore amounts to:

$$
\Gamma_{\mathrm{CR}}^{-}=\int_{-\infty}^{+\infty} d z \int_{B_{s}}^{B_{c}} \frac{d B_{J}}{w_{z}^{\text {in, },-}} j_{\text {in }}^{-}-\int_{-\infty}^{+\infty} d z \int_{B_{s}}^{B_{c}} \frac{d B_{J}}{w_{z}^{\text {out },-}} j_{\text {out }}^{+} .
$$

We now exchange the integral sums, which yields:

$$
\Gamma_{\mathrm{CR}}^{-}=\int_{B_{s}}^{B_{c}} d B_{J} \int_{-\infty}^{+\infty} d z\left(\frac{j_{\text {in }}^{-}}{w_{z}^{\text {in, },-}}-\frac{j_{\text {out }}^{+}}{w_{z}^{\text {out },-}}\right)
$$

This expression exclusively features upstream values of the specific angular momentum. As we consider the unsaturated horseshoe drag, we assume that it has same value as in the unperturbed disk. It is therefore independent of altitude, and can be taken out of the integral on $z$ :

$$
\Gamma_{\mathrm{CR}}^{-}=\int_{B_{s}}^{B_{c}} d B_{J}\left(j_{\text {in }}^{-} \int_{-\infty}^{+\infty} \frac{d z}{w_{z}^{\text {in, },-}}-j_{\text {out }}^{+} \int_{-\infty}^{+\infty} \frac{d z}{w_{z}^{\text {out },-}}\right) .
$$

We again apply Ertel's theorem with the ancillary function $\psi=z_{\text {in }}^{-}$, initial altitude of a fluid element, which gives:

$$
w_{z}^{\text {in, }-}=w_{z}^{\text {out, }-} \frac{\partial z_{\text {in }}^{-}}{\partial z_{\text {out }}^{-}}+w_{R}^{\text {out },-} \frac{\partial z_{\text {in }}^{-}}{\partial R_{\text {out }}} \approx w_{z}^{\text {out },-} \frac{\partial z_{\text {in }}^{-}}{\partial z_{\text {out }}^{-}},
$$

where we have again assumed that the radial tilt of the vortex tubes is negligible. We can therefore write:

$$
\int_{-\infty}^{+\infty} \frac{d z_{\text {out }}}{w_{z}^{\text {out },-}}=\int_{-\infty}^{+\infty} \frac{d z_{\text {out }}}{w_{z}^{\text {in,- }}} \frac{\partial z_{\text {in }}^{-}}{\partial z_{\text {out }}^{-}}=\int_{-\infty}^{+\infty} \frac{d z_{\text {in }}}{w_{z}^{\text {in, },-}} .
$$


We conclude that the integral $\int w_{z}^{-1} d z$ has same value in the upstream and downstream flow, far from the planet. The expression of this integral can be made slightly simpler by making use of the inverse of the vertical component of the PV, which we dubbed the load in an earlier paper (Masset \& Casoli 2010):

$$
l=\frac{\rho}{\zeta_{z}}
$$

and we denote with $L$ the vertical integral of the load:

$$
L=\int_{-\infty}^{+\infty} l(z) d z .
$$

We can now transform Eq. (37) into:

$$
\Gamma_{\mathrm{CR}}^{-}=\int_{B_{s}}^{B_{c}} d B_{J} L_{\mathrm{in}}\left(B_{J}\right)\left(j_{\mathrm{in}}^{-}-j_{\mathrm{out}}^{+}\right) .
$$

The jump of specific angular momentum during a U-turn, $\left(j_{\text {in }}^{-}-j_{\text {out }}^{+}\right)$, is expressed in term of the values in the unperturbed disk. It is a function of Bernoulli's invariant exclusively, which we denote $-\Delta j_{0}$. The horseshoe drag arising from the front part reads similarly:

$$
\Gamma_{\mathrm{CR}}^{+}=\int_{B_{s}}^{B_{c}} d B_{J} L_{\mathrm{out}}\left(B_{J}\right) \Delta j_{0},
$$

and the net corotation torque is:

$$
\Gamma_{\mathrm{CR}}=\int_{B_{s}}^{B_{c}} d B_{J}\left(L_{\mathrm{out}}-L_{\mathrm{in}}\right) \Delta j_{0}
$$

This integral, which only contains quantities of the unperturbed disk, can be evaluated using Eq. (21), which can be recast to lowest order as $\partial_{R} B_{J}=4 A_{p} B_{p} x$ and gives the relationship:

$$
B_{J}=B_{c}+2 A_{p} B_{p} x^{2},
$$

where $A=\frac{1}{2} r \partial_{r} \Omega$ and $B=\frac{1}{2 r} \partial_{r} j_{0}$ are respectively Oort's first and second constant. Changing our variable of integration to $x$, we are led to:

$$
\Gamma_{\mathrm{CR}}=8\left|A_{p}\right| B_{p}^{2} r_{p} \partial_{R} L_{0} x_{s}^{4} .
$$

Defining as in earlier works the dimensionless vortensity gradient at the planet's location as:

$$
\mathcal{V}=r_{p} \frac{\partial_{R} L_{0}}{L_{0}},
$$

and assuming that the flow's vorticity barely depends on altitude in the unperturbed disk, so that $L_{0} \approx \Sigma_{p} /\left(2 B_{p}\right)$, we are eventually led to:

$$
\Gamma_{\mathrm{CR}}=\frac{3}{4} \Sigma_{p} \Omega_{p}^{2} v x_{s}^{4},
$$

which is the exact same horseshoe drag expression as in the two dimensional case.

We conclude that the horseshoe dynamics in a threedimensional, globally isothermal disk, despite the complexity of the three dimensional flow, retain some of the simplicity of the two-dimensional case, firstly because the horseshoe region width is independent of altitude, which allows to use unambiguously a unique half width $x_{s}$, and secondly because the drag expression is the same as in the two-dimensional case, provided an adequate definition of the vortensity is used.
Eqs. (41), (42) and (47) show that the vortensity $V$ in a threedimensional disk must be defined as:

$$
V=\left[\int_{-\infty}^{+\infty}\left(\frac{\zeta_{z}}{\rho}\right)^{-1} d z\right]^{-1}
$$

\section{NUMERICAL SIMULATIONS}

\subsection{Mesh geometry and set up}

In order to illustrate and check the results of section 3, we have undertaken numerical simulations of a globally isothermal disk with a low mass planet embedded on a fixed circular prograde orbit, coplanar with the disk. We used for that purpose the public code FARGO3D ${ }^{5}$ (Benítez-Llambay \& Masset, submitted), with the setup p3diso, meant to describe a (locally or globally) isothermal gas in orbit around a pointlike mass, on a spherical mesh. The use of a spherical mesh may seem not adapted to the problem at hand, since we expect from section 3 a cylindrical symmetry for a number of variables, such as the azimuthal velocity or Bernoulli's invariant. In a similar manner, the horseshoe separatrices are expected to be cylinders. Our choice of a spherical mesh instead of a cylindrical one fulfills two purposes:

- owing to the strong flaring of a globally isothermal disk $\left(H \propto R^{3 / 2}\right)$, we avoid very empty regions at lower radius and high altitude.

- We can discard possible mesh effects if we find features with cylindrical symmetry.

Our mesh extends from $-\pi$ to $+\pi$ in azimuth, $0.65 r_{p}$ to $1.35 r_{p}$ in radius, and $\pi / 2-3 h_{p}$ to $\pi / 2$ in colatitude, where $h_{p}=0.05$ is the disk's aspect ratio at the orbital radius of the planet, so that $99 \%$ of the disk's mass at the planet location lies within the mesh. As we simulate only one hemisphere of the disk, we adopt reflecting boundary conditions at the equator. At high altitude we extrapolate the density and azimuthal velocity fields using the analytic profiles of Appendix A to fill the ghost zones, whereas we use symmetric boundary conditions on the radial velocity and antisymmetric boundary conditions on the velocity in colatitude. Our mesh size respectively in azimuth, radius and colatitude is $N_{\phi}=3290, N_{r}=367$ and $N_{\theta}=78$, with a uniform spacing, so that the cells are approximately cubic at $r=r_{p}$, with an edge length of $1.9 \cdot 10^{-3} r_{p}$. The planetary potential has the form:

$$
\Phi_{p}(\boldsymbol{r})=-\frac{G M_{p}}{\sqrt{\left|\boldsymbol{r}-\boldsymbol{r}_{p}\right|^{2}+\epsilon^{2}}},
$$

where $\epsilon$, a softening length used to avoid a divergence at the planet location, is set to $4 \cdot 10^{-3} r_{p}$, which is $8 \%$ of the pressure scale height, or two cell sizes. No viscosity is used in the calculations. The planet to star mass ratio is $q=10^{-5}$, so the planet has $8 \%$ of the thermal mass $h_{p}^{3} M_{\star}$.

The disk's initial conditions are given by $v_{r} \equiv v_{\theta} \equiv 0$, while the density and azimuthal velocity are given respectively by Eqs. (A18) and (A19). Damping boundary conditions (de ValBorro et al. 2006) are used in the radial direction only, over $10 \%$ of the radial extent of the mesh (i.e. over annuli of width $0.07 r_{p}$ ), both at the inner and outer edges. Each of our calculations is carried out over 20 orbital periods of the planet. We

\footnotetext{
${ }^{5}$ See http://fargo.in2p3.fr.
} 
do not use a temporal tapering of the planetary mass upon the planet insertion in the disk. The manner in which the planet is turned on can have an impact on the flow at larger time in two dimensions (Ormel et al. 2015a), since some vortensity can be created by shocks and trapped in a closed region around the planet, but no such region exists in three dimensions (Ormel et al. 2015b), and our setup does not have the resolution to capture processes that happen on a scale of the planetary Bondi radius.

Our fiducial calculation is one for which $\alpha=3 / 2$, which corresponds to a (nearly) vanishing vortensity gradient. Our background disk is therefore identical to that of Fung et al. (2015). The main differences are, in our case, a smaller planetary mass, and a much coarser resolution at the planet location.

In addition to this fiducial calculation, we have run ten others calculations in which we vary the vortensity gradient, defined by Eq. (48). Overall we have 11 runs with a vortensity gradient ranging from -1.5 to 1.5 by increments of 0.3 .

\subsection{Separatrix sheet and stagnation points}

We perform all our analyses at $t=20$ orbital periods of the planet, the date $t=0$ corresponding to the planet insertion in the disk.

We determine the position of the separatrices by integrating the path of fluid elements starting at a given colatitude and radius $r$, with azimuth $\phi= \pm 1$. The integration is carried out downstream for $\phi\left(r-r_{c}\right)>0$, and upstream otherwise. The trilinearly interpolated value of each component of the velocity field is used for the integration. For each colatitude, the starting radius of the widest horseshoe streamline is found with a dichotomic search to machine accuracy.

We represent on Fig. 2 the width of horseshoe region as a function of the distance to the midplane. As expected from section 3.2 , the width is nearly constant, to within a few percent, over the whole vertical extent of the computational domain, which covers three vertical scale heights. Also shown in this figure is the width of the horseshoe region from $2 \mathrm{D}$ calculations with different potential's softening lengths. We see that they match for a softening length of $0.65 H$. Interestingly, this value is comparable to the softening length required to match the two- and three-dimensional Lindblad torques (Masset 2002; Kley et al. 2012). These results are to be compared with those of Fung et al. (2015), who considered a marginally sub-thermal mass planet, and who find the horseshoe width reproduced by $2 \mathrm{D}$ calculations with a softening length $\sim 0.35 \mathrm{H}$. The average value of the front and rear widths measured in our fiducial run is:

$$
x_{s}=0.0147 r_{p}
$$

In order to determine the location of the stagnation points, we proceed as outlined in section 3.2 , i.e. by determining the manifolds of decreasing dimension obtained by imposing successively that $v_{\phi}=0$, then also $v_{r}=0$, and finally also $v_{\theta}=0$. Care must be taken in this procedure with the staggering of the velocity components in the FARGO3D code. Namely, for each of the $N_{\theta}$ conical slices of our mesh, we determine the location at which $v_{\phi}$ cancels out (the intersection of the corotation sheet with the cone $\theta=\theta_{k}$, with $\theta_{k}=\pi / 2-3 h_{p}(k+1 / 2) / N_{\theta}$ and $\left.k \in\left[0, N_{\theta}-1\right]\right)$. This location turns out to be unique, for our setup and resolution, and results in a function of $r$, for any given azimuth $\left(\phi_{i}\right)_{i \in\left[0, N_{\phi}-1\right]}$ : $r=r_{c}\left(\phi_{i}, \theta_{k}\right)$. Next we linearly interpolate $v_{r}$ at the locations $\left[\phi_{i}, r_{c}\left(\phi_{i}, \theta_{k}\right), \theta_{k}\right]$. For each value of $k$ (within each slice in co-

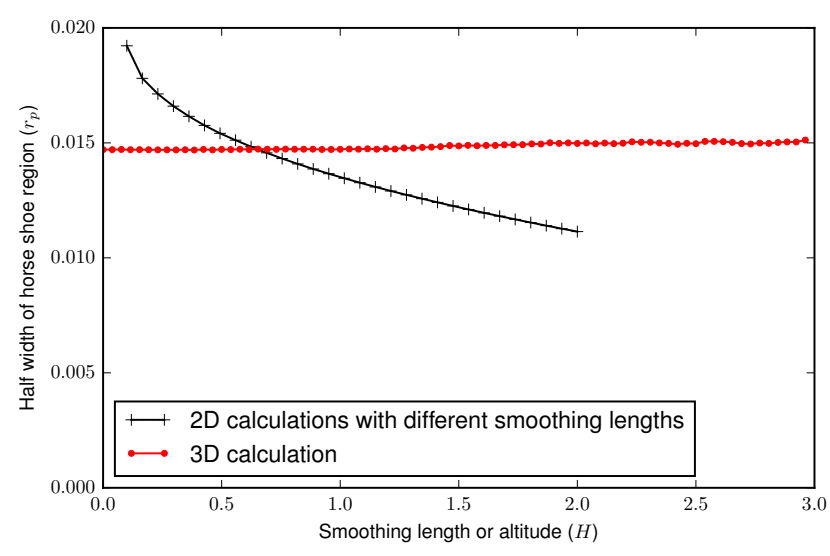

FIG. 2.- Width of the horseshoe region as a function of altitude $(\pi / 2-\theta) r_{p}$, expressed in pressure scale heights $H$. The value reported is the arithmetic mean of the width measured at $\phi=1$ and $\phi=-1$ (using a dichotomic search of the separatrices position; the dots represent the altitudes at which this search was performed). The black crosses show the width measured in auxiliary two-dimensional calculations, in which case the $x$-axis represent the softening length used for the potential, in units of $H$. This figure should be compared to figure 3 of Fung et al. (2015).

latitude), we thus construct a sequence $v_{r}^{i}$. We then seek the $\operatorname{root}(\mathrm{s})$ in $\phi$ of the piecewise linear function that has value $v_{r}^{i}$ for $\phi=\phi_{i}$. We thus obtain, for each slice in colatitude, the location at which the bilinearly interpolated values of the azimuthal and radial velocities simultaneously vanish. By varying $k$, we identify "filaments of horizontal stagnation", nearly vertical curves in which the azimuthal and radial velocities cancel out. These filaments are not streamlines, since they can have some tilt in azimuth and radius, whereas the velocity field on them is purely along the colatitude direction, by construction 6 There are not sets of stagnation points either, because in general $v_{\theta}$ does not vanish on these filaments, except at specific locations which are determined in our last step, which consists in constructing for each filament the sequence of $v_{\theta}^{k}$, the bilinearly interpolated values of $v_{\theta}$ where the filaments intersect the zone edges in colatitude. As previously, we then seek the roots of the piecewise linear function that coincides with $v_{\theta}^{k}$ for $\theta=\pi / 2-3 h_{p} k / N_{\theta}$. This gives us the location of the stagnation points, where the three velocity components simultaneously vanish. As a sanity check, we verify that the trilinearly interpolated value of each velocity component is indeed very small at the location of each stagnation point. We overall identify 96 stagnation points in our fiducial run at 20 orbits, most of them relatively far for the planet, especially in the tadpole regions. Of special interest is the filament that lies near the planet, almost at the intersection of the separatrix sheets. This filament contains three stagnation points: one at the midplane, and two others within the first pressure scale height.

We show in Fig. 3 the separatrix sheets of the horseshoe region. Although the streamlines of these sheets exhibit significant vertical motion toward stagnation points in the vicinity of the stagnation filament, as expected from the considerations of section 3.2, they never quite reach these points: we find that an accuracy higher than double precision would

\footnotetext{
${ }^{6}$ If they were streamlines, they would be circles centered on the star and contained in vertical planes, since their velocity would be exclusively along the colatitude direction. This is not possible because, by definition, they are contained in the corotation sheet, which has cylindrical symmetry. A stagnation filament depends on the coordinate system and does not have, contrary to the stagnation points, a physical meaning per se.
} 


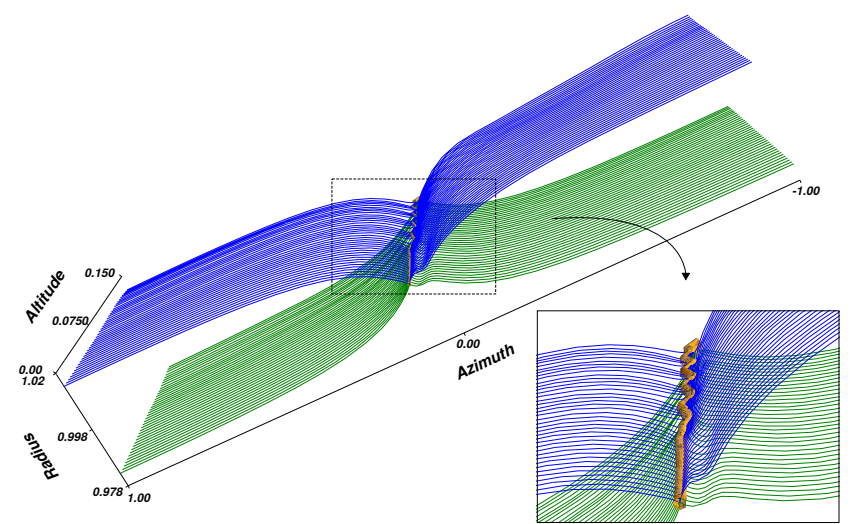

FIG. 3.- Three-dimensional view of the separatrices of the horseshoe region (widest horseshoe streamlines). The axes represent the cylindrical coordinates $(R, \phi, Z)$. For legibility purposes, the radial coordinate has been stretched by a factor of 2 , while the azimuthal coordinate has been compressed by a factor of 5 . The orange tube at the streamlines intersection represents a filament of horizontal stagnation, in which three stagnation points can be identified (see main text for details).

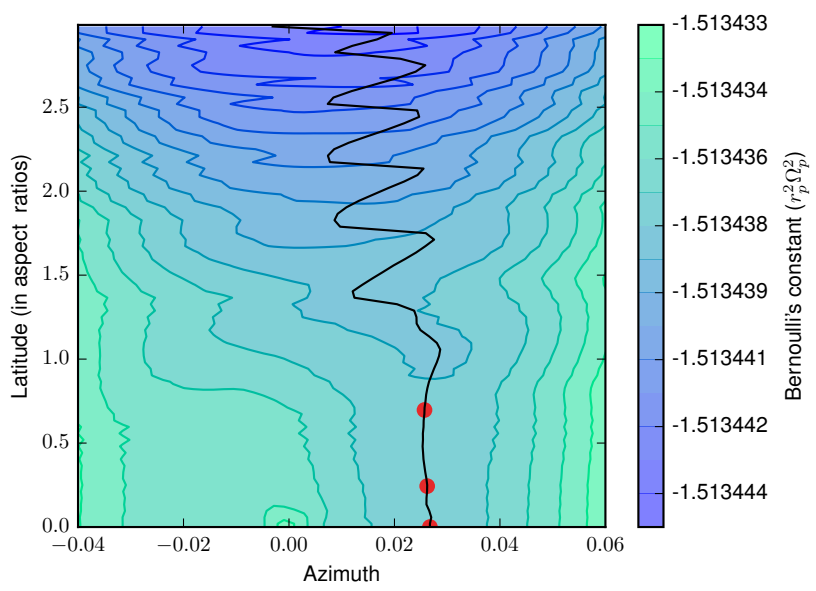

FIG. 4.- Bernoulli's invariant in the corotation sheet. The horizontal axis represents $\phi$ and the vertical axis represents $(\pi / 2-\theta) / h$. The map shown corresponds to the linearly interpolated value of Bernoulli's invariant at $r_{c}(\phi, \theta)$. The black line shows the filament of horizontal stagnation (see text for details), and the three red dots on this filament are stagnation points.

be required to better constrain the starting radius of a separatrix streamline that would reach the vicinity of the stagnation points. Our assertion of section 3.2 that Bernoulli's invariant is uniform on the separatrix sheets, and equal to its value at the corresponding stagnation point therefore needs to be examined in more detail.

\subsection{Bernoulli's invariant in our fiducial calculation}

Fig. 4 shows the value of Bernoulli's invariant in the corotation sheet, in the planet vicinity. The horizontal stagnation filament is shown in this figure. Bernoulli's invariant is found to vary by less than $10^{-6} r_{p}^{2} \Omega^{2}$, at the filament location, over the first pressure scale height vertically, and it decays by $6-7 \cdot 10^{-6} r_{p}^{2} \Omega_{p}^{2}$ over the next two scale heights. This overall variation is to be put in contrast to the drop of Bernoulli's invariant between corotation (at large distance from the planet) and separatrices, given by Eqs. (46) and (52), which amounts to $-8 \cdot 10^{-5} r_{p}^{2} \Omega_{p}^{2}$. The variation over the first scale-height is therefore at the percent level, whereas the variation at higher altitude is at most ten percent of the drop of Bernoulli's in-

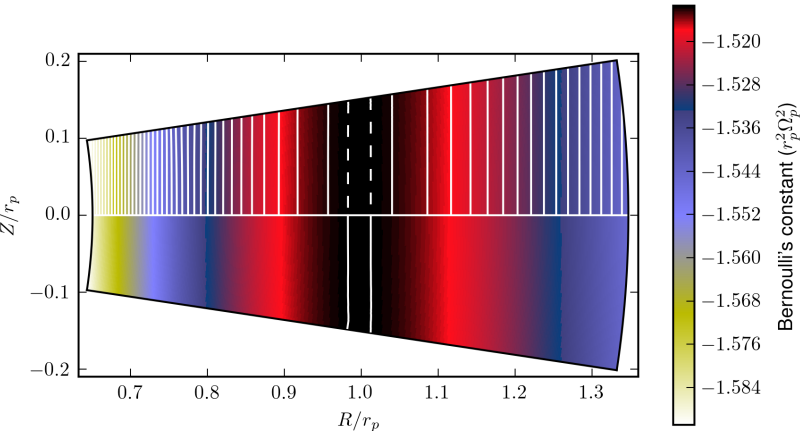

FIG. 5.- Meridional slice $(R, Z)$ showing isocontours of Bernoulli's invariant in the upper half, and the separatrix sheet in the lower half, at azimuth $\phi=+1 \mathrm{rad}$, for the whole computational domain. The dashed lines show isocontours of $B_{s}$, value measured for Bernoulli's invariant at the stagnation points (see text for details). As could be expected from Eq. 21, or Eq. 46, Bernoulli's invariant is maximal at corotation.

variant between corotation and separatrices. The value of Bernoulli's invariant at the three stagnation points on the filament are respectively, by order of increasing distance to the midplane:

$$
\begin{aligned}
& B_{s}^{1}=-1.51343737 r_{p}^{2} \Omega_{p}^{2} \\
& B_{s}^{2}=-1.51343732 r_{p}^{2} \Omega_{p}^{2} \\
& B_{s}^{3}=-1.51343750 r_{p}^{2} \Omega_{p}^{2}
\end{aligned}
$$

In order to better assess to which extent Bernoulli's invariant can be regarded as uniform over the separatrix sheets, we define $B_{s}=-1.5134374 r_{p}^{2} \Omega_{p}^{2}$, an average of the values quoted above, and we compare the isosurfaces $B_{J} \equiv B_{s}$ to the separatrix sheets. Fig. 5 shows a global view of a meridional slice of the disk. The upper half of the figure shows isocontours of Bernoulli's invariant at $\phi=1 \mathrm{rad}$. The lower half shows the separatrix sheets at same azimuth. The cylindrical symmetry of the separatrix sheet and iso-Bernoulli surfaces is readily apparent, and we find an excellent coincidence between the radii of the separatrices and the radii of the isosurfaces of $B_{s}$. Here we use a subscript $s$ to convey that $B_{J}$ has to be evaluated at a stagnation point. In section 3.3.2, we used a similar notation to represent the (unique) value of $B_{J}$ on a separatrix sheet. Our numerical results confirm our expectation of section 3.2 that both values coincide, hence it is legitimate to use the same notation $B_{s}$ for both values.

Fig. 6 shows a detailed comparison of the upstream separatrix distance to corotation with the distance of the isosurface $B_{J} \equiv B_{s}$. A nearly perfect agreement is found over the first pressure scale height, with residual errors far below the mesh resolution. It is likely that the trilinearly interpolated values of Bernoulli's invariant on the one hand, and of the velocity on the other hand, fulfill Eq. (14) with a high accuracy, but we have not investigated the reasons for this nearly perfect match in detail. Nonetheless, this remarkable agreement lends confidence in the fact that Bernoulli's invariant can be regarded as uniform on the separatrix sheet, and equal to the value $B_{s}$ that it has on the stagnation filament. The agreement, as can be seen in Fig. 6, is not so good at higher altitudes (albeit with a discrepancy between Bernoulli's isosurface and the separatrices still below the mesh resolution). This corresponds to the altitudes at which Bernoulli's invariant shows a small depar- 


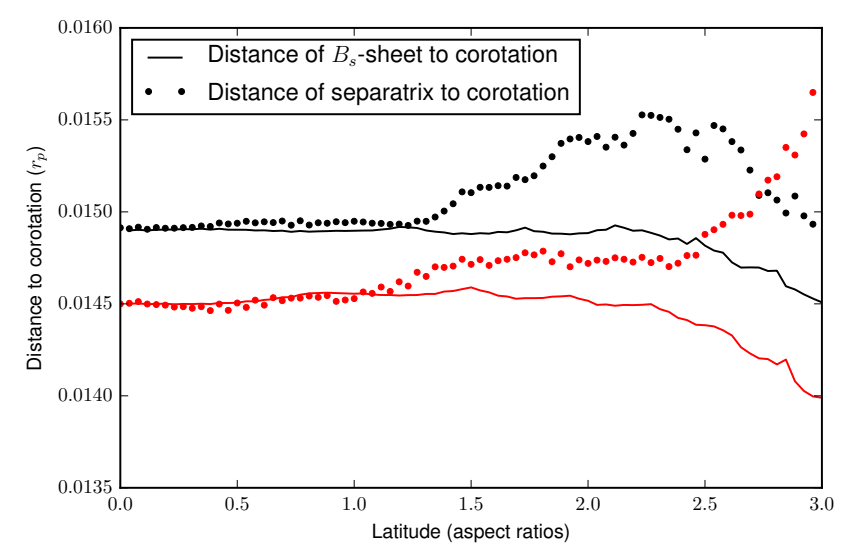

FIG. 6.- Detailed view of the upstream separatrix versus isosurface of Bernoulli's invariant, for the front part $(\phi=1 \mathrm{rad}$, in black) and the rear part $\left(\phi=-1 \mathrm{rad}\right.$, in red). The $x$-axis represents $(\pi / 2-\theta) / h_{p}$, and the $y$-axis the distance to corotation at constant colatitude, in semi-major axis of the planet.

ture from the uniform value that it has over the first pressure scale height (see Fig. 4), which suggests that the flow has not reached a fully steady state at higher altitudes 20 orbital periods after the insertion of the planet. Our streamline integration shows indeed that the high altitude fluid elements near the separatrix spend a considerable amount of time near the horizontal stagnation filament, where they execute significant vertical excursions at very small velocity (thereby contributing to homogenize Bernoulli's invariant along this filament.) We will come back to the timescale of high altitude U-turns in section 6.2, and we will see that they can last much longer than in the midplane. We note that we could not have taken a significantly larger time after the planet insertion to perform our analysis, because the first effects of phase mixing are expected around $t=40$ orbital periods for the horseshoe zone width that we measured.

We note in Fig. 4 that over the first pressure scale-height, the filament of horizontal stagnation is almost vertical. We could have defined stagnation filaments by the requirement $v_{R}=v_{\phi}=0$ (that is, the first two components of the velocity in a cylindrical coordinate system vanish). The filament thus defined could nearly be a streamline connecting the stagnation points, directly evidencing why they share same value of Bernoulli's invariant.

\subsection{Total torque and horseshoe drag}

Our following step is to check whether Eq. (49) gives a reliable estimate of the horseshoe drag. This expression requires the prior knowledge of $x_{s}$, the half-width of the horseshoe region. We use for this purpose the set of simulations that we performed with different vortensity gradients, and we assume that the width of the horseshoe region, as in the twodimensional case, does not sensitively depend on the vortensity gradient (Casoli \& Masset 2009) and is given by Eq. (52).

We show in Fig. 7 the value measured for the torque in our 11 calculations, as a function of the surface density slope, and we compare it to two analytical expressions: one of them is the total torque estimate obtained in the linear regime by TTW02, which reads:

$$
\Gamma_{\text {TTW02 }}=-(1.364+0.541 \alpha) \Sigma_{p} \Omega_{p}^{2} r_{p}^{4} q^{2} h_{p}^{-2},
$$

and the other one is the sum of the linear estimate of the Lindblad torque of TTW02, and the non-linear estimate of the corotation torque of Eq. 49, in which we set $V=\frac{3}{2}-\alpha$. The Lindblad torque estimate of TTW02 is:

$$
\Gamma_{\mathrm{TTW} 02}^{\mathrm{LR}}=-(2.340-0.099 \alpha) \Sigma_{p} \Omega_{p}^{2} r_{p}^{4} q^{2} h_{p}^{-2} .
$$

The value obtained in our calculations are much more compatible with the second estimate. This result extends to the threedimensional case the findings of Paardekooper \& Papaloizou (2009a) that the corotation torque eventually becomes nonlinear at all planetary masses, even those which are largely sub-thermal. In addition, it shows that the horseshoe drag formula of Eq. (49) gives an acceptable estimate of the non-linear corotation torque.

\subsection{Width of the horseshoe region}

The half width that we have found for the horseshoe region, given by Eq. (52), is only 3.5 times larger than the softening length. One can therefore wonder whether the horseshoe region could be substantially wider in the limit of a vanishing softening length. In order to answer this question, we have undertaken two additional calculations with the same setup as our fiducial one, except for the softening length, which was set respectively to $0.06 \mathrm{H}$ and $0.10 \mathrm{H}$, instead of our fiducial value of $0.08 \mathrm{H}$. We find that the horseshoe half-width displays a nearly linear relationship with the softening length, with a very small slope, and that the width extrapolated for a vanishing softening length is only $0.8 \%$ wider than in our fiducial calculation: $x_{s}=0.0148 r_{p}$.

This width is marginally smaller than the widely used estimate derived for two-dimensional disks with a softening length of $0.3 \mathrm{H}$ for the potential, which reads $x_{s}=$ $1.16 r_{p}(q / h)^{1 / 2}$ (Masset et al. 2006). Here, we find a slightly different numerical factor, about $10 \%$ smaller, for the halfwidth of the horseshoe region:

$$
x_{s}=1.05 r_{p} \sqrt{\frac{q}{h}} .
$$

These findings are consistent with those of Lega et al. (2015), who also observe a $10 \%$ smaller width for the horseshoe region than the standard two-dimensional estimate for subthermal mass planets.

\subsection{Relationship between perturbed effective potential and horseshoe width}

In the limit of a small planetary mass, the location of all stagnation points tend to the corotation of the unperturbed disk, since $v_{\phi}=R\left(\Omega_{0}-\Omega_{p}\right)+v_{\phi}^{\prime}$, where $v_{\phi}^{\prime}$ is the perturbed velocity due to the planet, which scales with $q$ when the planetary mass is sufficiently small. We restrict our discussion to this limiting case, which is tantamount to assuming that the corotation sheet is not significantly distorted by the introduction of the planet, at the location of the stagnation points. Denoting $\boldsymbol{r}_{s}$ the location of a stagnation point, we can write Bernoulli's constant at $\boldsymbol{r}_{s}$ prior to the planet insertion, which reads:

$$
B_{J, 0}=\Phi_{*}\left(\boldsymbol{r}_{s}\right)-\frac{1}{2} R_{c} \Omega_{p}^{2}+\eta_{0}\left(\boldsymbol{r}_{s}\right)=B_{c}
$$

and after the planet insertion, once a steady state is reached in the planet frame:

$$
B_{J}=\Phi_{*}\left(\boldsymbol{r}_{s}\right)+\Phi_{p}\left(\boldsymbol{r}_{s}\right)-\frac{1}{2} R_{c} \Omega_{p}^{2}+\eta\left(\boldsymbol{r}_{s}\right)=B_{s}
$$




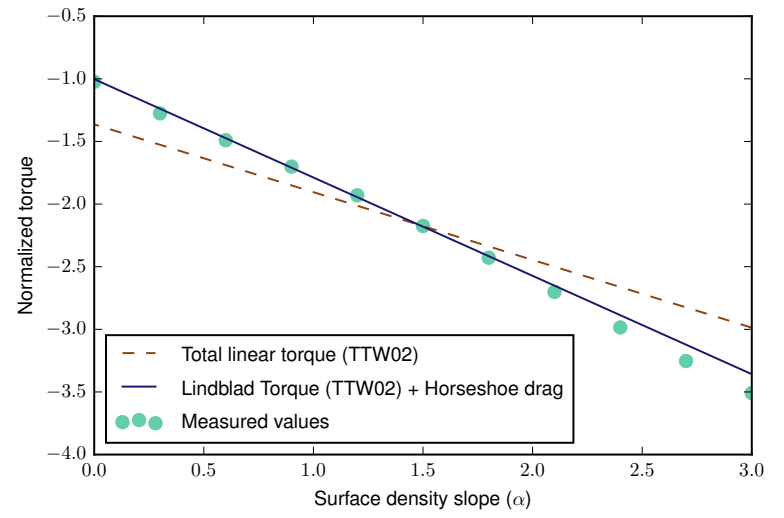

FIG. 7.- Total torque as a function of the surface density slope. The dashed line shows the total torque estimate by Tanaka et al. (2002), while the solid line shows the Lindblad torque estimate of Tanaka et al. (2002), plus the nonlinear corotation torque estimate provided by the horseshoe drag formula of Eq. [49, in which the width of the horseshoe region is provided by Eq. [55].

We therefore have:

$$
B_{s}-B_{c}=\Phi_{p}\left(\boldsymbol{r}_{s}\right)+\eta^{\prime}\left(\boldsymbol{r}_{s}\right),
$$

where $\eta^{\prime}=\eta-\eta_{0}$ is the perturbed enthalpy. Using a notation similar to Masset et al. (2006), we write:

$$
\tilde{\Phi}^{\prime}=\Phi_{p}+\eta^{\prime},
$$

which corresponds to the perturbed value of the effective potential of Eq. (10). Using Eq. (46), and specifying to the Keplerian case, for which $A_{p}=-3 \Omega_{p} / 4$ and $B_{p}=\Omega_{p} / 4$, we obtain:

$$
x_{s}=\frac{1}{\Omega_{p}} \sqrt{-\frac{8}{3} \tilde{\Phi}^{\prime}\left(\boldsymbol{r}_{s}\right)},
$$

which is the same as Eq. (12) of Masset et al. (2006). The value of the perturbed effective potential at the stagnation point is directly related to the width of the horseshoe region, and may be used to infer the latter from numerical simulations, without resorting to streamline analysis.

\subsection{Tilt angle}

In the derivation of Eq. (49), we had to assume that the vortex tubes had a small tilt angle with respect to the vertical direction in the downstream flow. We examine here how justified this assumption was. Fig. 8 shows the tilt angle in the radial direction (the tilt angle in the azimuthal direction does not feature in our torque derivation owing to the assumption that at large azimuthal distance the flow variables have a vanishing azimuthal derivative.) We see on this figure that slightly larger tilt angle values are systematically found in the downstream flow (inner side for $\phi=1 \mathrm{rad}$, outer side for $\phi=-1 \mathrm{rad}$ ). Nevertheless, the values observed are very small, largely below $10^{-2}$ over most of the horseshoe flow, especially over the first pressure scale height, whose contribution dominates the horseshoe drag. Our assumption was therefore largely satisfied for our fiducial run.

\subsection{Potential vorticity in the midplane}

We finally mention that, as expected, the PV is not conserved in the flow. This is illustrated in Fig. 9 where we see stripes in the downstream distribution of the vertical component of PV (at the midplane), which cannot be accounted for by advection of the initial distribution by the horseshoe flow.
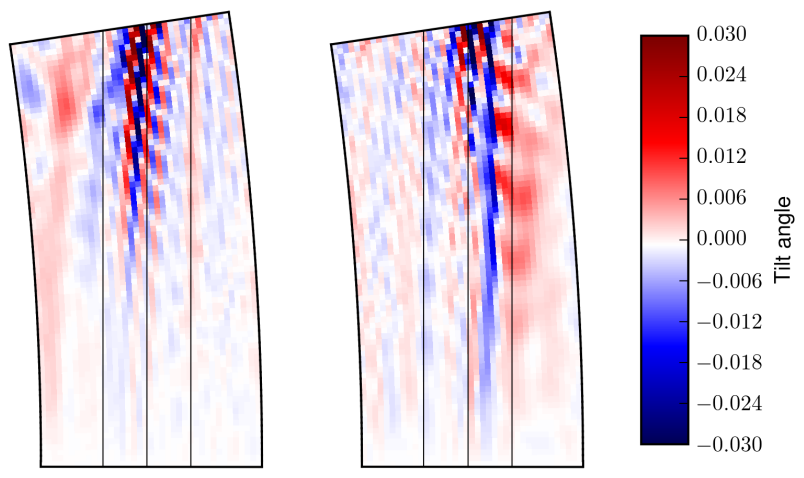

FIG. 8. - Tilt angle $\left(\zeta_{R} / \zeta_{z}\right)$ of the vorticity vector, at $\phi=+1 \mathrm{rad}$ (left) and $\phi=-1 \mathrm{rad}$ (right). The three thin vertical lines represent, from left to right, the inner separatrix, the corotation and the outer separatrix.

These stripes can be traced back to vertical oscillations of the material upon execution of their U-turn. Compression (expansion) of material along a vortex tube (i.e. essentially in the vertical direction, in our case) decreases (increases) the PV (as can be readily seen, for instance, from Ertel's theorem by taking for $\psi$ any curvilinear abscissa along the vortex tube).

\section{SUMMARY}

We have shown that the horseshoe region of a low-mass planet in a globally isothermal disk has a constant width with altitude and a cylindrical shape, in line with the recent results of Fung et al. (2015) for nearly thermal mass planets. We interpret this fact as the consequence of $a$ ) the conservation of Bernoulli's invariant, $b$ ) the fact that this constant must be uniform over the whole separatrix sheet (both boundaries of the horseshoe region), and $c$ ) the fact that Bernoulli's invariant does not depend on the altitude, but only on the cylindrical radius, in unperturbed globally isothermal disks. We note that in our numerical results, the horseshoe width is constant with altitude within a few percent, over the three scale lengths covered by our computational domain, and within one percent over the first pressure scale height. In contrast, the results of Fung et al. (2015) show a weak bulge at the $10 \%$ level at the disk midplane. It is yet to be determined whether this difference arises from the different masses (Fung et al. (2015) consider a nearly thermal mass, whereas we examined here the flow around a planet with only $8 \%$ of the thermal mass), or whether it arises from the transient horseshoe flow unveiled by Fung et al. (2015), that the analysis exposed here does not resolve. In any case, the results of Fung et al. (2015) are indicative of some disruption of the horseshoe separatrix near the midplane, since otherwise, as we have shown here, the separatrix sheets should have a strictly cylindrical shape. We further discuss this in section 6.4 .

In addition, we find that the horseshoe drag in threedimensional, globally isothermal disks, given by Eq. (49), has same expression as in two-dimensional disks, when expressed in terms of the width of the horseshoe region. In particular, the horseshoe drag is found to scale with the vortensity gradient. Our analysis of section 3.3 shows that, in a three-dimensional disk, the vortensity should be defined by Eq. (50).

The three-dimensional case therefore essentially retains the simplicity of the two-dimensional case. We find that the halfwidth of the horseshoe region, for sub-thermal mass planets, 

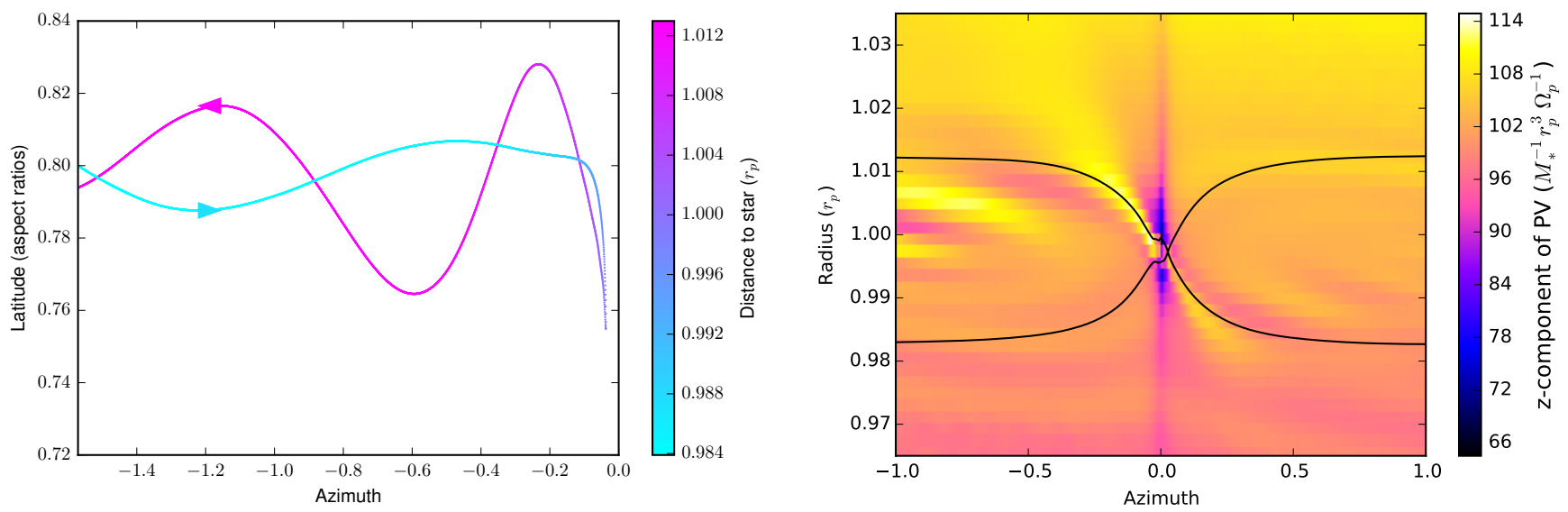

FIG. 9.- Horseshoe streamline near the separatrix, as seen from the star (left) and PV in the midplane (right). The color of the streamline on the left plot represents the distance to the star. The cyan part is the upstream one, while the purple part is downstream. The streamline decays toward the planet at the tip of the U-turn, as it tends to go toward the uppermost stagnation point of Fig. 4 It then emerges at a different altitude and oscillates vertically in the downstream flow. One can see on the right plot the imprint of these oscillations in the downstream flow, at the disk midplane, as the yellow and purple stripes. The black line shows the intersection of the separatrix with the midplane.

has the form given by Eq. (55), which can be recast, for an arbitrary adiabatic index $\gamma$, as:

$$
x_{s}=1.05 \gamma^{-1 / 4} r_{p} \sqrt{\frac{q}{h}} .
$$

This result is in good agreement with the recent findings of Lega et al. (2015), who also find that the width of the horseshoe region in three-dimensional situations is $\sim 10 \%$ smaller than previously envisioned from two-dimensional calculations with the customary value $\epsilon=0.3 H$ for the softening length of the potential. One consequence of this somewhat unexpected result is discussed in section C. Incidentally, we find that the value of the softening length that leads to twodimensional horseshoe regions with the width of Eq. (61) is $\epsilon=0.65 H$, a value close to that required to match the Lindblad torques of two- and three-dimensional disks.

\section{DISCUSSION}

\subsection{Comparison to the linear corotation torque}

Using Eq. (49) and (55), we can recast the non-linear corotation torque expression as:

$$
\Gamma_{\mathrm{CR}}=0.88 \mathcal{V} \Sigma_{p} \Omega_{p}^{2} r_{p}^{4} q^{2} h_{p}^{-2}
$$

Assuming that to lowest $\operatorname{order} \mathcal{V}=3 / 2-\alpha$, we have:

$$
\Gamma_{\mathrm{CR}} \approx(1.32-0.88 \alpha) \Sigma_{p} \Omega_{p}^{2} r_{p}^{4} q^{2} h_{p}^{-2} .
$$

This expression is to be compared with the linear corotation torque of TTW02, which reads:

$$
\Gamma_{\mathrm{CR}}^{\operatorname{lin}} \approx(0.976-0.64 \alpha) \Sigma_{p} \Omega_{p}^{2} r_{p}^{4} q^{2} h_{p}^{-2},
$$

showing that the non-linear corotation torque is $\sim 1.36$ times larger than the linear corotation torque. This statement is reminiscent of the two-dimensional case where a similar ratio is found between the non-linear and linear estimates of the corotation torques (Masset \& Casoli 2010, Paardekooper et al. 2011).

The linear estimate of TTW02 vanishes for $\alpha=1.525$, slightly above the value $3 / 2$ that one would naively expect. The vortensity gradient in a globally isothermal disk is actually not 3/2- $\alpha$. Using Eq. A19, we can show that it has the expression:

$$
\mathcal{V}=\frac{3}{2}\left(1+\frac{3}{2} h^{2}\right)-\left(1-\frac{3}{2} h^{2}\right) \alpha,
$$

where we neglect terms of order $h^{4}$ and above. The vortensity gradient therefore vanishes for $\alpha=3 / 2+(9 / 2) h^{2}$ (which in our fiducial disk is $\alpha=1.511$ ) and so does the non-linear corotation torque, which scales exactly with the vortensity gradient in our analysis.

\subsection{On the width at the midplane and at higher altitude}

One might have expected the width of the horseshoe region to decrease at higher altitudes, since the gas there is subjected to a reduced gravity from the planet, compared to the midplane. Similarly, one might have expected the width in the midplane to be similar to the width of the two-dimensional case in the limit of a vanishing softening length, since in this plane the motion is two-dimensional. This two-dimensional width, however, is much larger than the width reported here: instead of the value given by Eq. 61), it is $\sim 1.7 r_{p} \sqrt{q / h}$ (Paardekooper \& Papaloizou 2009b, Ormel 2013). Therefore two questions arise: why is the horseshoe region so narrow at the midplane, and why is it so wide at large altitudes ?

\subsubsection{Width at the midplane}

Eq. (60) shows that the width of the horseshoe region is determined by the value of the perturbed effective potential, given by Eq. (59), at the stagnation point. The well of perturbed effective potential is much shallower than the gravitational potential well of the planet, as the latter is partially filled with enthalpy (which is why the horseshoe region is much narrower than predicted by the restricted three body problem). In the $3 \mathrm{D}$ case, the density at the midplane differs from the density in a purely two-dimensional case with same velocity field because of the vertical motion above the midplane, which can compress the gas or allow it to expand. The difference of perturbed enthalpy between the three-dimensional case and a two-dimensional case with same resolution and parameters is shown in Fig. 10. This difference is also the difference of perturbed effective potentials between the two cases, since the planetary potential is the same in the two- and threedimensional cases. In the vicinity of the orbit, this difference 


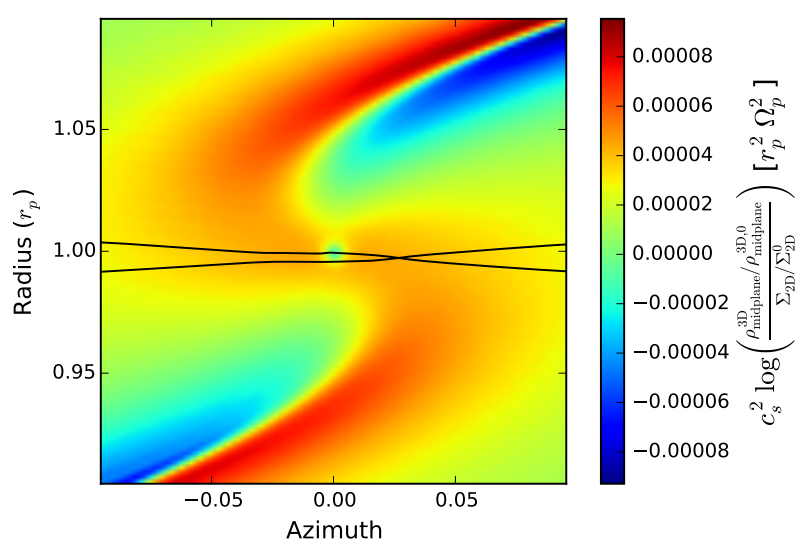

FIG. 10.- Difference of perturbed enthalpy between the three-dimensional case (at the midplane) and a two-dimensional case with same parameters. A positive value is found for $r \sim r_{p}$. Incidentally, we note an enthalpy excess upstream of the spiral wake, followed by a deficit downstream, compatible with a convergence of the fluid elements toward the midplane as they arrive at the wake, followed by an expansion.

is positive, resulting from the trend of the streamlines to bend toward the midplane. The effective potential well is therefore shallower in the 3D case than in the 2D case, and the horseshoe region is consequently narrower. The actual difference of the horseshoe width between the $2 \mathrm{D}$ and $3 \mathrm{D}$ cases cannot be deduced from Fig. 10 because the stagnation point can be at a different location in each case, but the order of magnitude of the enthalpy excess in the 3D case is compatible with a sizable reduction of the horseshoe width with respect to the two-dimensional case.

\subsubsection{Width at high altitude}

As we mentioned in section 4.3 , the high altitude fluid elements originating from the vicinity of the separatrix linger near the stagnation filament, taking thereby a large amount of time to perform their U-turn. This effect is responsible for maintaining a sizable width at higher altitudes. The time it takes to perform a horseshoe U-turn near the midplane is only a few orbital periods of the planet (Baruteau \& Masset 2008), but it has to take more time at higher altitude for the following reason: the planetary torque is smaller than its midplane value, but a fluid element located near the separatrix eventually exchanges with the planet the same amount of angular momentum as that of a fluid element at the same cylindrical radius in the midplane, because the horseshoe region has the same width at all altitudes. The reduction factor can be large in our fiducial case, since the typical azimuth along the stagnation filament, $\phi \sim 0.02$, is small compared to the latitude ( $0.15 \mathrm{rad})$ of the highest fluid elements, which indicates that the time it takes to execute a U-turn at higher altitude is large compared to that required near the midplane.

\subsection{Considerations on saturation}

This paper has focused on the unsaturated horseshoe drag. A detailed study of the saturation properties will be presented elsewhere. We can however anticipate here the following two points:

- As pointed out in section 6.2.2 the widest horseshoe Uturns are performed more slowly at large altitude. In a two-dimensional situation, saturation occurs as a result of phase mixing because streamlines located at different distances of corotation have different libration times. In a three-dimensional situation, streamlines at same distance to corotation but at different altitudes also have different libration times 7 We can therefore anticipate that the torque oscillations should exhibit a pattern different from those of two-dimensional disks (see e.g. Masset \& Casoli 2010), and that the torque should relax faster toward its asymptotic value as a result of enhanced phase mixing.

- In the evaluation of the saturated torque value, the parameter $x_{s}$ (half-width of the horseshoe region) plays a very important role. The torque asymptotic (or saturated) value depends on a competition between libration (which tends to cancel the torque, as it flattens the gradients of vortensity and entropy across corotation) and diffusive processes (viscous and thermal diffusion, which tend to restore the large scale gradients and the unsaturated torque value). Of these two processes, the one with the shortest timescale will win the competition. The ratio of the diffusive to the libration time scales is proportional to $x_{s}^{3}$ (Masset 2001; Masset \& Casoli 2010, Paardekooper et al. 2011), hence the ultimate torque value depends very sensitively on $x_{s}$. An accurate estimate of $x_{s}$ such as the one we provide here is therefore crucially important in correctly evaluating the degree of saturation of the corotation torque. Masset \& Casoli (2010) suggested that the width of the horseshoe region was expected to depend on the altitude, and that a value at low altitude, where most of the mass resides, could be used to determine the torque value. In view of the results reported here, this suggestion is clearly not adequate, and it leads, not surprisingly, to corotation torque values too saturated and therefore largely sub-estimated (Bitsch \& Kley 2011). Regardless of the improvements required for three-dimensional torque formulae, we show in appendix C that using for $x_{s}$ the value given by Eq. (61) improves considerably the torque estimate of Masset \& Casoli (2010) and essentially reconciles it with other estimates (Paardekooper et al. 2011).

\subsection{Relationship with the flow at sub-Bondi scale}

Recent work has highlighted the characteristics of the flow at the scale of Bondi's radius $r_{B}=G M_{p} / c_{s}^{2}$ and below (Ormel et al. 2015b), and the potential impact of this flow on the horseshoe drag (Fung et al. 2015). Ormel et al. (2015b) show that the flow can enter the Bondi sphere at high altitude and exit near the midplane (in shear dominated configurations) or enter the Bondi sphere near the midplane and exit at larger altitude (in headwind dominated configurations). In the former case, Fung et al. (2015) have found, for a slightly sub-thermal mass planet, that the material expelled near the midplane can reach and cross the horseshoe's boundary. They call this flow the transient horseshoe flow, as it involves fluid elements that participate in a unique close encounter with the planet. This flow destroys the horseshoe boundary (near the midplane), invalidating our assumption of the existence of a critical surface: streamlines of different origins merge near the edge of the downstream horseshoe flow. Our work completely disregards these potentially very important effects, owing to its

${ }^{7}$ The libration time is only a factor $h^{-1}$ larger than the U-turn time for subthermal mass planets (Baruteau \& Masset 2008). If the U-turn time at higher altitude is several times larger than at the midplane, it may then represent a sizable fraction of the overall libration time. 
moderate resolution. As noted by Fung et al. (2015), the huge resolution needed to resolve the flow within the Bondi sphere for planetary masses as low as those considered here is beyond computational tractability on present day platforms, at least for single mesh calculations, even with non-uniform resolution. The problem of the impact of the flow at sub-Bondi scales on the horseshoe drag should be tackled by means of nested mesh calculations, which is a forthcoming feature of the code that we used in this paper. Fung et al. (2015) find that the total torque in their calculation is still negative, but reduced in magnitude with respect to the expected value of the linear Lindblad torque. They perform a horseshoe drag analysis of their outcome by summing the mass flow rate, weighted by the angular momentum jump (which they determine by a streamline integration), over the whole set of horseshoe streamlines. They find that the contribution of the transient horseshoe flow to the torque is negligible, and that there exists a net positive corotation torque due to the standard horseshoe flow, in spite of the disk being barotropic with a vanishing vortensity gradient. It is noteworthy that they find a non-vanishing contribution at all altitudes (see their Fig. 15), even above the first pressure scale height, where the flow most resembles the situation that we described here, with a columnar structure and well defined separatrix sheets. There are ingredients neglected in our analysis that could play an important role, such as the radial tilt of vortex tubes upon U-turns, or the non-conservation of Bernoulli's constant if shocks are present in the planet vicinity. The impact of the flow at subBondi scale on the planetary torque clearly requires further work, in which such ingredients should probably be incorporated.

Extensions of the present work could also consider the impact of the radial temperature gradient (in so-called locally isothermal disks) or the role of the entropy gradient in flows with an energy equation (in so-called adiabatic disks). The entropy gradient is known to have a major impact on the corotation torque in three-dimensional disks (Paardekooper \& Mellema 2008; Lega et al. 2015), but so far its action on the coorbital flow and the generation mechanism of the entropyrelated torque have been studied only in two-dimensional disks (Masset \& Casoli 2009, Paardekooper et al. 2010).

We thank Bertram Bitsch for kindly providing us with the simulation data described in appendix C. We thank Jeffrey Fung, Pawel Artymowicz and Yanqin $\mathrm{Wu}$ for insightful discussions on the transient horseshoe drag. We also thank Gloria Koenigsberger, Jeffrey Fung, Elena Lega and Bertram Bitsch for constructive comments on a first draft of this manuscript, and an anonymous referee for constructive comments which improved the quality of this manuscript. F. Masset acknowledges support from CONACyT grant 178377. Pablo Benítez-Llambay acknowledges financial support from CONICET. We thank Ulises Amaya Olvera, Reyes García Carreón and Jérôme Verleyen for their assistance in setting up the GPU cluster on which the calculations presented here have been run.

\section{APPENDIX}

\section{PROFILES OF DISKS IN ROTATIONAL AND HYDROSTATIC EQUILIBRIUM}

The standard procedure that consists in adopting a Gaussian vertical profile of the disk density is only approximate, and has a poor accuracy at altitudes higher than the disk's pressure scale height. Here we derive exact relations for the disk's density and azimuthal velocity profiles, under conditions slightly more general than those considered in sections 3 and 4 . We assume that the sound speed is a power law of the spherical radius:

$$
c_{s}^{2}(r)=\left(c_{s}^{0}\right)^{2}\left(\frac{r}{r_{0}}\right)^{-\beta} .
$$

where $r_{0}$ is an arbitrary radius at which the sound speed is $c_{s}^{0}$. Such disks are often qualified of locally isothermal. The aspect ratio has the radial dependence:

$$
h(r)=\frac{c_{s}(r)}{v_{K}(r)} \propto r^{(1-\beta) / 2}
$$

where $v_{K}(r)=\sqrt{G M_{\star} / r}$ is the circular Keplerian velocity at distance $r$ from the central mass. We call flaring index the exponent $f$ of the power law given by Eq. (A2):

$$
f=\frac{1-\beta}{2}
$$

For the globally isothermal disks considered in the main part of this paper, we have $\beta=0$ and $f=1 / 2$. The equations that determine the rotational and vertical equilibria of the disk are respectively, in spherical coordinates 8

$$
-\frac{\partial_{r}\left(\rho_{0} c_{s}^{2}\right)}{\rho_{0}}+\frac{v_{\phi}^{2}}{r}-\frac{G M_{\star}}{r^{2}}=0
$$

and

$$
-\frac{1}{r} \frac{\partial_{\theta}\left(\rho_{0} c_{s}^{2}\right)}{\rho_{0}}+\frac{v_{\phi}^{2}}{r} \cot \theta=0
$$

If we denote $L=\log \left(\rho_{0} / \rho_{00}\right), m=v_{\phi}^{2} / c_{s}^{2}, u=-\log (\sin \theta), v=\log \left(r / r_{0}\right)$ and $K=G M_{\star} /\left[c_{s}\left(r_{0}\right)^{2} r_{0}\right]$, we can transform Eq. A5 into:

$$
\partial_{u} L+m=0
$$

\footnotetext{
${ }^{8}$ In this appendix only, $v_{\phi}$ denotes the azimuthal velocity in a non-rotating frame.
} 
and Eq. (A4) into

$$
-\beta+\partial_{v} L-m+K \exp (-2 f v)=0,
$$

where we have made use of the assumption that the sound speed only depends on the spherical radius. Deriving Eq. A6 with respect to $v$ and Eq. A7] with respect to $u$, we are led to

$$
\partial_{v} m+\partial_{u} m=0,
$$

from which we infer:

$$
\partial_{u^{k}}^{k} m=(-1)^{k} \partial_{v^{k}}^{k} m .
$$

The rotational equilibrium in the midplane reads, from Eq. (A4):

$$
m(u=0, v)=-\beta-\xi+K \exp (-2 f v),
$$

hence, for any $k \geq 1$, we have in the midplane $(u=0)$ :

$$
\partial_{v^{k}}^{k} m=(-2 f)^{k} K \exp (-2 f v),
$$

so that, by virtue of Eq. A9, we have, also in the midplane:

$$
\partial_{u^{k}}^{k} m=(2 f)^{k} K \exp (-2 f v),
$$

from which we can reconstruct the value of $m$ at an arbitrary height above the midplane:

$$
m(u, v)=[\exp (2 f u)-1] K \exp (-2 f v)+m(u=0, v)=-\beta-\xi+K \exp [2 f(u-v)],
$$

which specifies the field of rotational velocity. The density field is found by integrating Eq. (A6], which yields:

$$
L=L_{\mathrm{eq}}+(\beta+\xi) u-K \frac{e^{-2 f v}}{2 f}[\exp (2 f u)-1],
$$

where the eq subscript denotes the midplane value. Using the more conventional notation, Eqs. (A13) and (A14) read respectively:

$$
v_{\phi}(r, \theta)=v_{K}(r)\left[(\sin \theta)^{-2 f}-(\beta+\xi) h^{2}\right]^{1 / 2},
$$

where $v_{K}(r)$ is the circular Keplerian velocity at distance $r$ from the central mass, and

$$
\rho_{0}(r, \theta)=\rho_{\mathrm{eq}}(r)(\sin \theta)^{-\beta-\xi} \exp \left[h^{-2}\left(1-\sin ^{-2 f} \theta\right) / 2 f\right] .
$$

For a "flat" disk, in which the temperature is inversely proportional to the radius ( $\beta=1$ and $f=0$ ), the integration of Eq. A6) eventually yields:

$$
\rho_{0}(r, \theta)=\rho_{\mathrm{eq}}(\sin \theta)^{-\beta-\xi+h^{-2}},
$$

For globally isothermal disks, Eqs (A16) and A15) can be recast respectively as:

$$
\begin{gathered}
\rho(r, \theta)=\rho_{\mathrm{eq}} \sin ^{-\xi} \theta \exp \left[h^{-2}\left(1-\frac{1}{\sin \theta}\right)\right] \\
v_{\phi}^{2}(r, \theta)=\frac{G M_{\star}}{r \sin \theta}-\xi c_{s}^{2}=\frac{G M_{\star}}{R}-\xi c_{s}^{2} .
\end{gathered}
$$

The rotational velocity is therefore independent of the altitude at a given cylindrical radius in globally isothermal disks. Finally, for $z / R \ll 1$, we have $u \approx \frac{1}{2}(z / R)^{2}$, hence Eq. A14 can be recast in the following approximate form, when $f u \ll 1$ :

$$
L \approx L_{\mathrm{eq}}-\frac{1}{2} h\left(r_{0}\right)^{-2}\left(\frac{r}{r_{0}}\right)^{-2 f}\left(\frac{z}{R}\right)^{2}
$$

where use has been made of the relationship $h^{-2} \gg|\xi+\beta|$. As a consequence, we recover the well known approximation:

$$
\rho_{0}(z) \approx \rho_{\mathrm{eq}} \exp \left(-z^{2} / 2 H^{2}\right)
$$

from which we can infer the relationships

$$
\Sigma_{0}(r)=\sqrt{2 \pi} \rho_{\mathrm{eq}} H
$$

and

$$
\alpha=\xi-1-f
$$




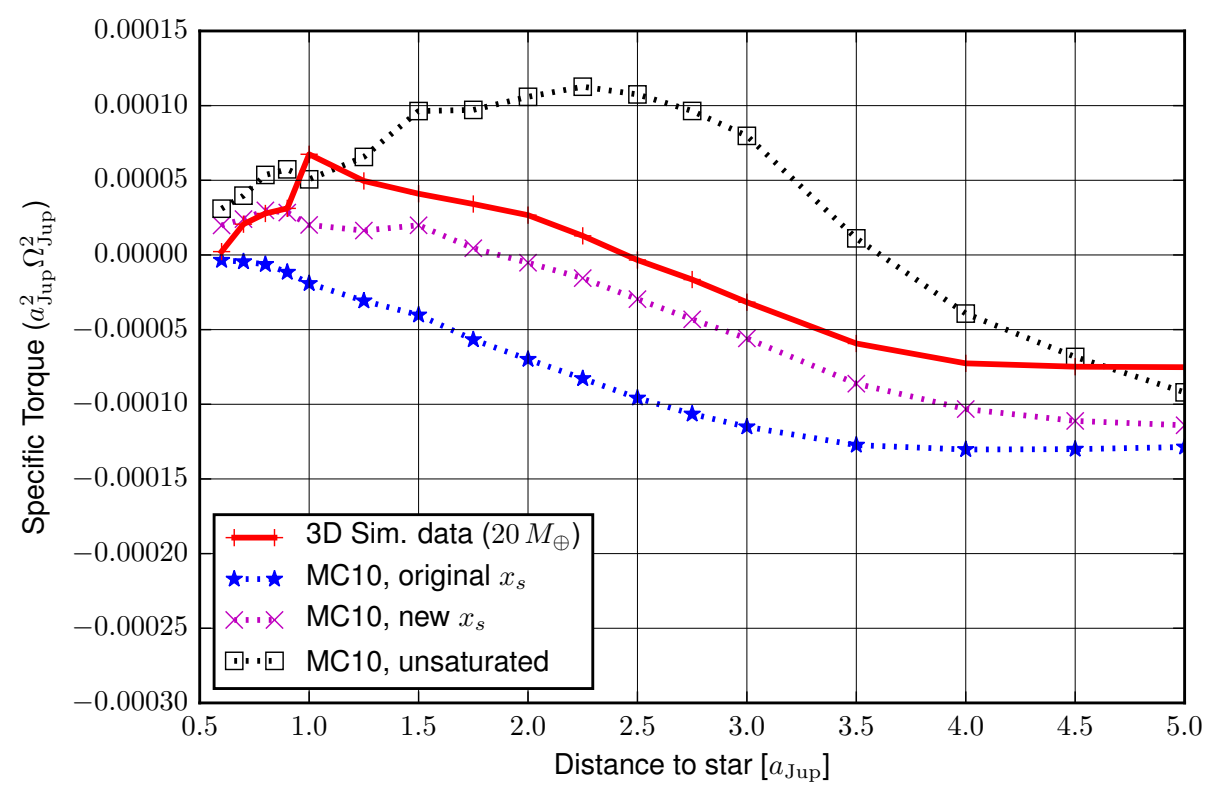

FIG. 11. - Total torque estimate with the value of $x_{s}$ found in this work (magenta curve), compared to old estimate for an over-wide horseshoe region (blue). This figure should be compared to Fig. 3 of BK11. The simulation data (red curve) have been kindly provided by B. Bitsch.

\section{UNIQUENESS OF BERNOULLI'S INVARIANT ON A GIVEN SEPARATRIX SHEET}

We use reductio ad absurdum to show that, in steady state, a separatrix sheet cannot be connected to two stagnation points with different values of Bernoulli's invariant. We assume a horseshoe separatrix sheet to be connected to two stagnation points with values of Bernoulli's invariant $B_{1}$ and $B_{2} \neq B_{1}$. The value of Bernoulli's invariant on the separatrix sheet is piecewise constant: it is equal to $B_{1}$ on the streamlines connected to the first point, and it is equal to $B_{2}$ on the streamlines connected to the second point. It is therefore discontinuous at the critical streamline separating these two domains. Eq. (15) shows that the only term that can be discontinuous in the expression of Bernoulli's invariant is the kinetic energy (since the gravitational potential is continuous in space, and so is the enthalpy in a barotropic fluid, away from shocks.) At large distance from the planet, where the radial and vertical velocities are negligible, the azimuthal velocity must be discontinuous across the critical streamline. The radial balance, given by Eq. (6), reads:

$$
\frac{v_{\phi}^{2}}{R}=-\partial_{R} \eta-\partial_{R} \Phi .
$$

The potential gradient being continuous, the discontinuity of $v_{\phi}$ must be borne by $\partial_{R} \eta$. Denoting $z_{c}$ the altitude of a point $C$ on the critical streamline, we have $\left.\partial_{R} \eta\right|_{z_{c}^{+}} \neq\left.\partial_{R} \eta\right|_{z_{c}^{-}}$. This precludes the continuity of $\eta$ on any neighborhood of $C$, which is impossible. Our initial assumption is therefore impossible and the separatrix sheet cannot be connected to points with different values of Bernoulli's invariant. Numerical experiments confirm this expectation: discontinuities in the azimuthal velocity along the vertical direction would correspond to singular sheets of radial vorticity, which are not observed.

\section{EFFECT OF $X_{S}$ ON THE SATURATED TORQUE VALUE}

We reproduce here the comparison performed by Bitsch \& Kley (2011, hereafter BK11) between simulations of a $20 M_{\oplus}$ planet embedded in a three-dimensional radiative disk, and the torque formula of Masset \& Casoli (2010, hereafter MC10). The unique amendment to the original formula is that we use for $x_{s}$ the value provided by Eq. (61), instead of the larger value suggested by MC10 in their Eq. (157). Fig. 11] shows that the resulting new estimate is much closer than the original one to the simulation data. A perfect agreement should not be expected for the large planetary mass (typically thermal) considered here, since we use a formula valid only for largely sub-thermal mass planets. The width $x_{s}^{\prime}$ of the horseshoe region of a $20 M_{\oplus}$ planet is likely larger than the estimate given by Eq. 61. The horseshoe drag scales with $x_{s}^{4}$, and the saturation degree, for a partially saturated torque as is the case here, scales with $x_{s}^{-3}$. The torque value should therefore be underestimated by a factor $x_{s}^{\prime} / x_{s}$ (where $x_{s}$ is given by Eq. 61, consistent with our estimate being systematically under the simulation data. The near compensation of these two effects (horseshoe width boost for thermal mass planets, largely compensated for by an increased degree of saturation) has recently been discussed by Lega et al. (2015), who note that torque formulae for sub-thermal mass planets give reasonable torque estimates for planetary masses largely beyond their domain of validity.

\section{REFERENCES}

Baruteau, C., \& Masset, F. 2008, ApJ, 672, 1054

Bitsch, B., \& Kley, W. 2011, A\&A, 536, A77

Casoli, J., \& Masset, F. S. 2009, ApJ, 703, 845

D'Angelo, G., Kley, W., \& Henning, T. 2003, ApJ, 586, 540 de Val-Borro, M., Edgar, R. G., Artymowicz, P., Ciecielag, P., Cresswell, P., D’Angelo, G., Delgado-Donate, E. J., Dirksen, G., Fromang, S., Gawryszczak, A., Klahr, H., Kley, W., Lyra, W., Masset, F., Mellema, G., Nelson, R. P., Paardekooper, S.-J., Peplinski, A., Pierens, A., Plewa, T., Rice, K., Schäfer, C., \& Speith, R. 2006, MNRAS, 695 
Ertel, H. 1942, Meteorol. Z., 59, 277

Fung, J., Artymowicz, P., \& Wu, Y. 2015, ApJ, 811, 101

Goodman, J., \& Rafikov, R. R. 2001, ApJ, 552, 793

Guilet, J., Baruteau, C., \& Papaloizou, J. C. B. 2013, MNRAS, 430, 1764

Kley, W., Müller, T. W. A., Kolb, S. M., Benitez-Llambay, P., \& Masset, F. 2012, A\&A, 546, A99

Lega, E., Morbidelli, A., Bitsch, B., Crida, A., \& Szulágyi, J. 2015, MNRAS, 452, 1717

Masset, F. S. 2001, ApJ, 558, 453

-. 2002, A\&A, 387, 605

Masset, F. S., \& Casoli, J. 2009, ApJ, 703, 857

-. 2010, ApJ, 723, 1393

Masset, F. S., D'Angelo, G., \& Kley, W. 2006, ApJ, 652, 730

Ogilvie, G. I., \& Lubow, S. H. 2002, MNRAS, 330, 950

Ormel, C. W. 2013, MNRAS, 428, 3526

Ormel, C. W., Kuiper, R., \& Shi, J.-M. 2015a, MNRAS, 446, 1026

Ormel, C. W., Shi, J.-M., \& Kuiper, R. 2015b, MNRAS, 447, 3512
Paardekooper, S., Baruteau, C., Crida, A., \& Kley, W. 2010, MNRAS, 401, 1950

Paardekooper, S., Baruteau, C., \& Kley, W. 2011, MNRAS, 410, 293

Paardekooper, S.-J., \& Mellema, G. 2008, A\&A, 478, 245

Paardekooper, S.-J., \& Papaloizou, J. C. B. 2009a, MNRAS, 394, 2283 -. 2009b, MNRAS, 394, 2297

Rafikov, R. R. 2002, ApJ, 572, 566

Schubert, W., Ruprecht, E., Hertenstein, R., Nieto Ferreira, R., Taft, R., Rozoff, C., Ciesielski, P., \& Hung-Chi, K. 2004, Meteorologische Zeitschrift, 13, 527

Tanaka, H., Takeuchi, T., \& Ward, W. R. 2002, ApJ, 565, 1257

Ward, W. R. 1986, Icarus, 67, 164

Ward, W. R. 1991, in Lunar and Planetary Institute Conference Abstracts, 1463 